\title{
Acknowledgment to Reviewers of Cells in 2021
}

\section{Cells Editorial Office}

Citation: Cells Editorial Office. Acknowledgment to Reviewers of Cells in 2021. Cells 2022, 11, 477. https://doi.org/10.3390/cells11030477 Published: 29 January 2022

Publisher's Note: MDPI stays neutral with regard to jurisdictional claims in published maps and institutional affiliations.

Copyright: () 2022 by the author. Licensee MDPI, Basel, Switzerland. This article is an open access article distributed under the terms and conditions of the Creative Commons Attribution (CC BY) license (https://creativecommons.org/licenses/by/4.0/).

MDPI AG, St. Alban-Anlage 66, 4052 Basel, Switzerland

Rigorous peer-reviews are the basis of high-quality academic publishing. Due to the great efforts of our reviewers, Cells was able to maintain its standards for the high quality of its published papers. Thanks to the contribution of our reviewers, in 2021, the median time to first decision was 16 days and the median time to publication was 40 days. Among the reviewers who reviewed for Cells in 2021, the following five have been selected to receive the "Cells 2021 Outstanding Reviewer Awards" for the timeliness and quality of their review reports in 2021:

Bonam, Srinivasa Reddy from Université de Paris, Paris, France Homma, Takujiro from Yamagata University, Yamagata, Japan Hwang, In Koo from Seoul National University, Seoul, Korea Kobeissy, Firas from University of Florida, Gainesville, FL, USA Rebelo, Sandra from University of Aveiro, Aveiro, Portugal

The editors also express their gratitude and recognition to the following reviewers for their precious time and dedication, regardless of whether the papers they reviewed were finally published:

Abate, Andrea

Abd El-Aziz, Tarek Mohamed

Abdelalim, Essam M.

Abdel-Daim, Mohamed M.

Abdolmaleki, Parviz

Abe, Kenji

Abe, Kuniya

Abend, Michael

Aboubakar, Frank

Abraham, Dietmar

Åbrink, Magnus

Absalon, Sabrina

Acosta-Alvear, Diego

Acquasaliente, Laura

Adachi, Yasushi

Adadi, Parise

Adamek-Urbańska, Dobrochna

Adjaye, James

Adunyah, Samuel E.

Aedo Abadie, Jorge Eduardo

Afanassieff, Marielle

Afferni, Claudia

Afonso, Philippe

Afrin, Sadia
Agbarya, Abed

Aguayo Paul, Sebastián

Agudo, Judith

Aguilera-Tejero, Escolastico

Agulló-Ortuño, María Teresa

Ahearne, Mark

Ahluwalia, Balpreet

Ahmad, Faraz

Ahmad, Khurshid

Ahmadian, M. Reza

Ahn, Jaegyoon

Aiello, Sistiana

Ajiro, Masahiko

Akash, Sajid

Akihiko, Oka

Akutsu, Hidenori

Alaimo, Alessandro

Alani, Eric

Alarcón, Balbino

Albani, Diego

Albert, Antony

Alberti, Elena

Alberti, Paola

Albonici, Loredana 
Alcántara-Ortigoza, Miguel Angel

Aldaz, Marcelo

Aldrich, Melissa B.

Aldskogius, Håkan

Alessandrini, Federica

Alexander, Francis Borgio J.

Alexy, Tamas

Algar, Elizabeth

Al-Ghadban, Sara I.

Alhadidi, Qasim

Al-Haj-Zen, Ayman

Ali, Syed Azmal

Alibardi, Lorenzo

Alieva, Irina Borisovna

Allodi, Silvana

Almad, Akshata

Al-Massadi, Omar

Almeida, David R. P.

Almeida, Marisa

Almerico, Anna Maria

Al-Mubarak, Bashayer R.

Alonso, Javier

Alonso, Juan Carlos

Alonso, Miguel A.

Alpers, Charles

Alpízar, Yeranddy Aguiar

Alsaab, Hashem

Altamura, Concetta

Altomonte, Jennifer

Álvarez Martín, Javier

Álvarez-Mercado, Ana I.

Alvarez-Rodriguez, Manuel

Alvarez-Silva, Marcio

Alves, C. Henrique

Alves, Lysangela R.

Alviano, Francesco

Amadoro, Giuseppina

Amaral, Flavio A.

Amaral, Isabel F.

Amasheh, Salah

Ambrósio, António F.

Ameele, Jelle Van Den

Amin, Kawa

Amorim, Christiani

Ampofo, Emmanuel

Amthor, Helge

Ana, Juan-García

Anderson, Peter M.

Anderson, Sean A. S.

Anderson, Stacey E.

Andón, Fernando Torres
Andre, Helder

Andreev, Yaroslav

Andreeva, Elena

Andreotti, Amy

Angelastro, James

Angeletti, Andrea

Angelidi, Angeliki

Angelini, Annalisa

Angelini, Corrado

Angelova, Angelina

Angelova, Plamena R.

Anghel, Larisa

Anghileri, Elena

Angrand, Pierre-Olivier

Angrisani, Nina

Ansari, Rais

Antinozzi, Cristina

Antonopoulou, Smaragdi

Antony, Benny S. Eathakkattu

Antunes, Celia M.

Anwar, Mumtaz

Aparecida Rainho, Claudia

Apicella, Antonio

Apolloni, Savina

Apostolova, Nadezda

Appenzellar-Herzog, Christian

Aragonés, Julián

Araki, Toshiyuki

Arata, Toshiaki

Araujo, Arturo

Arber, Caroline

Aréchaga-Ocampo, Elena

Arellanes-Robledo, Jaime

Arias, Jonathan

Arif, Tasleem

Armaiz, Guillermo

Armant, Olivier

Armelin-Correa, Lucia Maria

Arnaud, Jacquel

Arndt, Claudia

Arosio, Paolo

Arranz, Amaia

Arrebola, Francisco A.

Arrigo, Cicero

Arroba, Ana I.

Arsenijevic, Yvan

Arthur, Connie

Arufe, María C.

Asaftei, Sebastian

Asahara, Shunichiro

Asakawa, Haruhiko 
Ashton, Trent

Asier, González

Askjaer, Peter

Aspatwar, Ashok

Aspenström, Pontus

Assunção-Miranda, Iranaia

Astigarraga, Itziar

Astruc, Thierry

Athanasiou, Labrini V.

Athanassiou, Panagiotis

Atkin-Smith, Georgia

Attanasio, Chiara

Attri, Kuldeep

Auburger, Georg

Audrito, Valentina

Augustine, Josy

Augustyniak, Daria

Auranen, Annika

Aurrand-Lions, Michel

Aust, Gabriela

Austin, Shane

Avidor-Reiss, Tomer

Ávila, Julio

Avila, Matias

Avni, Orly

Avril, Tony

Awad, Fawaz

Awatade, Nikhil

Aylon, Yael

Ayroldi, Emira Maria

Azarnia Tehran, Domenico

Azhar, Mohamad

Azimzadeh, Omid

Aziriova, Silvia

Aziz, Faisal

Azuma, Kotaro

Babić Leko, Mirjana

Babiychuk, Eduard

Bacete, Laura

Bacher, Ulrike

Backer, Ronald A.

Bácskay, Ildikó

Bae, Chang-Hyu

Baer, Patrick C.

Baez-Nieto, David

Bagalkot, Tarique

Baharoglu, Zeynep

Bahmad, Hisham

Baig, Mohammad

Bailis, Adam M.

Bailly, Maryse
Baiocco, Giorgio

Baj, Andreina

Baj-krzyworzeka, Monika

Balbi, Carolina

Balbi, Matilde

Baldanzi, Gianluca

Balduini, Walter

Balestrieri, Barbara

Balla, András

Balla, Jozsef

Balogh, Enikő

Baloyannis, Stavros J.

Balsinde, Jesus

Baltanás, Fernando C.

Balzamino, Bijorn Omar

Bamburg, James

Banerjee, Hirendranath

Banning, Antje

Bar, Eli E.

Barabutis, Nektarios

Barak, Boaz

Baran, Jaroslaw

Baranov, Maksim V.

Barbalho, Sandra Maria

Bárbara, Paranhos Coelho

Barbaric, Ivana

Barbieri, Federica

Barbosa Bessa, Theolis

Bardag-Gorce, Fawzia

Bardi, Giuseppe

Barghout, Samir

Barichello, Tatiana

Barili, Valeria

Barkan, Daniel

Barnstable, Colin

Baron, Byron

Baron, Martin

Bar-Or, David

Barosova, Hana

Barr, Kelli L.

Barraco, Nadia

Barre, François Xavier

Barreiro Iglesias, Antón

Barrera-Chimal, Jonatan

Barrientos, Antonio

Barrientos, Gabriela

Barrott, Jared J.

Bar-Shavit, Rachel

Barshtein, Gregory

Bartke, Andrzej

Barton, Samantha K. 
Bartova, Eva

Barvik, Ivan

Baskaran, Rathinasamy

Bassi, Anna Maria

Basso, Lilian

Basta-Kaim, Agnieszka

Bastarache, Julie

Basu, Devraj

Bathula, Chandra Sekhar

Batissoco, Ana Carla

Battiato, Alfio

Battistelli, Michela

Bauer, Johann

Baufreton, Jérôme

Bayrak, Ömer Faruk

Beaujean, Nathalie

Beaumatin, Florian

Becciolini, Andrea

Beck, Jessica A.

Becker, Doreen

Becskei, Attila

Bednarek, Andrzej K.

Begni, Veronica

Behbahani, Homira

Beheshti, Afshin

Beißert, Tim

Beksac, Meral

Bélanger, RichardI R.

Beliakova-Bethell, Nadejda

Beling, Antje

Belizario, José Ernesto

Bellavia, Daniele

Bellelli, Roberto

Bellezza, Ilaria

Bello, Luca

Bellomo, Francesco

Belluzzi, Elisa

Belo, José António

Belosludtsev, Konstantin

Bel'skaya, Lyudmila

Beltrán Frutos, Ester

Belvedere, Raffaella

Ben Abderrahim, Mohamed Ali

Bencúrová, Elena

Bendjennat, Mourad

Benedetti, Antonio

Benesch, Matthew

Benesch, Matthew G. K.

Benfaremo, Devis

Bennardo, Luigi

Bennett, David
Bennett, James P.

Bentivegna, Angela

Berenyiova, Andrea

Berezin, Alexander

Bergamini, Carlo

Bergantini, Laura

Berge, Derk Ten

Berglund, Alix

Berg-Sorensen, Kirstine

Bergstrom, Kirk

Berman, Jason N.

Bermejo, Rodrigo

Bernabeu Aznar, Maria

Bernal, Juan A.

Bernardes, Emerson S.

Bernardi, Simona

Bernardo, Antonietta

Bernasconi, Sergio

Bernatik, Ondrej

Bernhardt, Ingolf

Bernstein, Sanford I.

Berthelot, Laureline

Bertolin, Giulia

Bertozzi, Giuseppe

Bertram, Kirstie

Bertrand, Fred

Bertrand, Pascale

Bertzbach, Luca D.

Besnard, Valérie

Besse, Andrej

Besse, Lenka

Betancourt, Miguel

Betran, Esther

Beutner, Gisela

Bezu, Lucillia

Bhandoola, Avinash

Bharath, Leena P.

Bhardwaj, Rajesh

Bhat, Jaydeep

Bhati, Kaushal Kumar

Bhatia, Divya

Bhattarai, Niina

Bhattarai, Prabesh

Bhawal, Ujjal Kumar

Bhere, Deepak

Bhute, Vijesh J.

Biagini, Giuseppe

Białek, Agnieszka

Biamonte, Flavia

Bianchi, Serena

Bianchini, Francesca 
Bianchini, Rodolfo

Bianconi, Fortunato

Biazzi, Elisa

Bidel, L. P. R.

Bidone, Tamara Carla

Bieberich, Erhard

Bielak-Żmijewska, Anna

Bieńkiewicz, Andrzej

Bierhoff, Holger

Bierings, Marc B.

Bijak, Michał

Bikov, András

Bilban, Martin

Binotto, Gianni

Bird, David

Bironaité, Daiva

Birtele, Marcella

Birukov, Konstantin

Bisti, Silvia

Biswas, Debolina

Bizzarri, Mariano

Björn, Lars-Olof

Blagojevic Zagorac, Gordana

Blagojević, Jelena

Blanc, Etienne

Blank, Ulrich

Blanquet, Véronique

Blas-Garciá, Ana

Blasiak, Janusz

Blazer-Yost, Bonnie L.

Blazquez, Ana-Belen

Blázquez-Castro, Alfonso

Blondonnet, Raiko

Blumer, Michael

Blyszczuk, Przemyslaw

Bocchinfuso, Gianfranco

Boehme, Karen A.

Boeldt, Derek S.

Boer, Cindy G.

Boersma, Arnold

Bogaard, Harm Jan

Bogdanov, Ivan V.

Bogdanov, Patricia

Bogie, Jeroen F. J.

Bognár, Zsófia

Boguszewska, Karolina

Boguta, Magdalena

Boia, Raquel

Boisvert, François-Michel

Boitani, Carla

Boix, Loreto
Boldogh, Istvan

Boldorini, Renzo

Bollini, Sveva

Bolomsky, Arnold

Bonanad, Santiago

Bonato, V. L.

Bonaventura, Jiř́i

Bonazzi, Vanessa F.

Boncompagni, Simona

Bonfanti, Luca

Bongrand, Pierre

Bönig, Halvard

Bonner, Caroline

Bonnet, Crystel

Booth, Stephanie A.

Boraschi, Diana

Bordin, Luciana

Borek, Sławomir

Borgese, Nica

Bornens, Michel

Borriello, Adriana

Borriello, Giovanna

Borutinskaite, Veronika

Bosáková, Michaela

Bosque, Alberto

Boto, Tamara

Botta, Elena

Bottai, Daniele

Boucher, Dave

Bouckaert, Julie

Bouitbir, Jamal

Boulares, Hamid

Bouley, Richard

Boulton, Michael E.

Bouma, Gerrit Jerry

Bourgoin, Sylvain

Bourmeyster, Nicolas

Bousset, Luc

Boussin, François

Boussios, Stergios

Boutte, Cara C.

Bovolenta, Paola

Boye, Kjetil

Bozgeyik, Ibrahim

Bozic, Josko

Brabek, Jan

Braconi, Daniela

Braganza, Andrea

Brakenhielm, Ebba

Branca, Ferdinando

Branca, Jacopo Junio Valerio 
Brancaccio, Andrea

Brancaleoni, Valentina

Braun, Ralf

Braun, Thorsten

Bravo, Susana B.

Brechbuhl, Heather

Bredeloux, Pierre

Breitfeld, Jana

Brenmoehl, Julia

Brennan, Meadhbh

Brenner, Catherine

Bresson-Bepoldin, Laurence

Brestic, Marian

Brindley, Paul

Brini, Marisa

Brink, Peter

Brinton, Margo

Brisson, Lucie

Brizzi, Maria Felice

Brocca, Stefania

Brockmeyer, Phillipp

Brodie, Chaya

Brodie, Graham

Broers, Jos LV

Brogi, Simone

Brooks, Philip John

Bros, Matthias

Bross, Peter

Brown, Dennis

Brown, Nelson

Brown, Nicholas

Brozovic, Anamaria

Bruinsma, Bote

Brukman, Nicolas

Brunelli, Silvia

Brunet De La Grange, Philippe

Brunetti, Dario

Brünnert, Daniela

Bruno, Giovanni L.

Brunoni, Federica

Bruria, Ben-Zeev

Bruschini, Luca

Brustovetsky, Nickolay

Brzezińska-Błaszczyk, Ewa

Brzóska, Edyta

Bucci, Cecilia

Büch, Thomas

Buchta, Christoph

Buchwalow, Igor Borisowitsch

Bucolo, Claudio

Budai-Szűcs, Mária
Bueno, Marta

Buentello-Volante, Beatriz

Buerger, Claudia

Buffo, Annalisa

Bugarcic, Andrea

Bugno-Poniewierska, Monika

Buitrago-Molina, Laura Elisa

Buraschi, Simone

Burchell, Joy

Burch-Smith, Tessa

Burckhardt, Christoph

Burdzinska, Anna

Burelle, Yan

Burga, Laura N.

Burger, Michael C.

Burgos, Miguel

Burkhead, Jason

Burmistrz, Michal

Burokas, Aurelijus

Burster, Timo

Busch, Karin

Bustelo, Xosé R.

Butler, Daniel S. C.

Butoi, Elena

Buzhdygan, Tetyana P.

Caaveiro, Jose

Caballero, Raúl Pérez

Caballo, Mar Carrión

Cabrera-Serrano, Macarena

Cabrini, Giulio

Caccamo, Daniela

Cacci, Emanuele

Caceres, Sara

Cacopardo, Ludovica

Cadamuro, Massimiliano

Caeiro, Maria Filomena

Caetano, Guilherme

Caffarelli, Elisa

Cahill, Michael

Cahill, Paul

Cai, Kai

Caiazzo, Massimiliano

Cairrão, Elisa

Calabrese, Vittorio

Calabresi, Laura

Calabresi, Paolo

Caldwell, R. William

Calhim, Sara

Calì, Tito

Caligo, Adelaide

Calina, Daniela 
Calistri, Arianna

Calka, Jaroslaw

Callaini, Giuliano

Calle-Guisado, Violeta

Calò, Annalisa

Calvier, Laurent

Calzia, Enrico

Camaioni, Antonella

Camargo, Mariana

Cambria, Maria Teresa

Cameron, Donald

Cammarata, Francesco

Cammarata, Giuseppe

Cammas, Florence

Camougrand, Nadine

Campa, Carlo Cosimo

Campagnolo, Marta

Campana, Luca Giovanni

Campbell, Grant R.

Campbell, Lia H.

Campbell, Nicole K.

Campello, Elena

Campinho, Marco

Campo, Gianluca

Campo, Giuseppe Maurizio

Campos-Toimil, Manuel

Campuzano, Althea

Campuzano, Oscar

Canaan, Stephane

Canberk, Sule

Candiani, Simona

Canela, Enric I.

Canettieri, Gianluca

Cangiano, Alberto

Canhoto, Jorge

Canosa, Stefano

Cano-Sarabia, Mary

Cantelmo, Anna Rita

Cantz, Tobias

Cao, Hongnan

Cao, Jingli

Capanni, Cristina

Caplan, Michael

Capovilla, Maria

Cappadocia, Laurent

Cappuccilli, Maria

Carafa, Vincenzo

Carai, Andrea

Caramujo, Maria José

Carbone, Emilio

Carbone, Marco
Carceller, Hector

Cardador-Martínez, Anaberta

Cardona, Fernando

Cardoso, Danon Clemes

Cardoso, Susana

Carducci, Federica

Caretta, Antonio

Cargnoni, Anna

Cariboni, Anna

Carina, Valeria

Carleo, Alfonso

Carlin, Cathleen R.

Carlton, Jeremy

Carman, Christopher V.

Carmo, Alexandre

Carmody, Ruaidhri

Carmona, Ju

Carnell, George

Carnevale Neto, Fausto Carnevale

Carnevale, Roberto

Carrascal, Livia

Carrassa, Laura

Carraway, Kermit L.

Carrella, Sabrina

Carreras, Joaquim

Carrie, Chris

Carrión, Mar

Carter, Edward P.

Carter, Wayne

Carullo, Gabriele

Caruntu, Constantin

Caruso, Donatella

Carvajal-Vergara, Xonia

Carvalho, Andreia N.

Carvalho, Juliana Lott

Carvalho, Pedro

Carvelli, Lucia

Carver, Wayne

Casadei, Nicolas

Casado-Díaz, Antonio

Casar, Berta

Casas, François

Casco, Víctor Hugo

Casini, Giovanni

Cassinerio, Elena

Castandet, Benoît

Castoldi, Angela

Castro-Munozledo, Federico

Castro-Torres, Rubén Darío

Catt, Sally

Cattane, Nadia 
Causse, Sebastien. Z.

Cava, Claudia

Cavaillé, Jerome

Cavalcanti, Marcos Fernando Xisto Braga

Cavazzoni, Andrea

Cavodeassi, Florencia

Cawley, Niamh

Ceccato-Antonini, Sandra Regina

Cedeño, David L.

Čedíková, Miroslava

Celada, Antonio

Celec, Peter

Celi, Alessandro

Cenariu, Mihai

Cenci, Giovanni

Cenni, Vittoria

Cens, Thierry

Centrone, Mariangela

Cerasuolo, Marianna

Cerdeira, Cláudio Daniel

Cermak, Lukas

Černá, Marie

Černý, Martin

Cervetto, Chiara

Cha, Hyuk-Jin

Chaiyasut, Chaiyavat

Chakrabarti, Jayati

Chakrabarti, Mrinmay

Chakrabarti, Rajarshi

Chakrabarty, Samit

Chakraborty, Paramita

Chalas, Renata

Chan, Ben C. L.

Chan, K. H. Katie

Chan, Sunny S. K.

Chandra, Abhishek

Chang, Chuang-Rung

Chang, Ling-Chu

Chang, Nai-Jen

Chang, Nan-Shan

Chang, Sheng-Nan

Chang, Shu-Chun

Chang, Theresa Li-Yun

Chang, Wei-Chao

Chang, Wei-Ju

Chang, Wen-Wei

Chang, Yih Hsin

Chao, Wang

Chapuis, Julien

Charbonnier, Jean-Baptiste

Charbord, Pierre
Charest, Pascale

Charriaut-Marlangue, Christiane

Chateigner-Boutin, Anne-Laure

Chatla, Kamalakar

Chatterjee, Madhumita

Chatterjee, Shambhabi

Chaudhry, Rasul

Chauhan, Bharesh

Chavanelle, Vivien

Chávez-Calvillo, Gabriela

Chcialowski, Andrzej

Chéli, Yann

Chen, Ceshi

Chen, Cheng-Chang

Chen, Chien Lun

Chen, Chien-Chin

Chen, Chien-Liang

Chen, Chi-Shuo

Chen, Chung-Hwan

Chen, Chung-Ming

Chen, Chun-Jung

Chen, Chun-Liang

Chen, Der-Yuan

Chen, Dongbao

Chen, Guan

Chen, Guoxun

Chen, Hungwen

Chen, Jianxiong

Chen, Jingshu

Chen, Ju

Chen, Kok Siong

Chen, Lihua

Chen, Lizhen

Chen, Mei-Yu

Chen, Ming

Chen, Shen-Liang

Chen, Shih-Heng

Chen, Shuen-Ei

Chen, Shukun

Chen, Wei

Chen, $\mathrm{Xi}$

Chen, Y. T.

Chen, Yih-Fung

Chen, Ying

Chen, Yu-Chih

Chen, Yung-Chia

Cheng, Chunchia

Cheng, Hung-Chi

Cheng, Juei-tang

Cheng, Kuang-Hung

Cheng, Po-Ching 
Cheng, Shih-Lung

Cheng, Shi-Yuan

Cheng, Tsung-Lin

Cheng, Xian-wu

Cheong, Heesun

Chernoff, Jonathan

Cherry, Jonathan

Chesler, Naomi

Chesnokova, Vera M.

Chester, Adrian $\mathrm{H}$.

Cheung, Wai W.

Cheynier, Remi

Chi, Hsiang-Cheng

Chi, Wei

Chiarella, Emanuela

Chibon, Frederic

Chikamori, Fumio

Childs, Gwendolyn Vaughn

Chimienti, Guglielmina

Chinen, Javier

Chiramel, Abhilash

Chiu, Ingming

Chmielewski, Michal

Cho, Chunghee

Cho, Hyunsoo

Cho, Myeong-Cheoul

Cho, Seong Beom

Cho, Young-Eun

Choi, Dae Eun

Choi, Hae Woong

Choi, Hueng-Sik

Choi, Kyung-Chul

Choi, Minee

Choi, Youkyung H.

Choi, Young Whan

Chorianopoulou, Styliani N.

Chorostecki, Uciel

Chou, Feng-Cheng

Choubey, Divaker

Choudhuri, Subhadip

Chow, Poming

Christ, Torsten

Christian, Preußer

Christiane, Stehmann

Christoforou, Nicolas

Chroni, Angeliki

Chu, Xiangping

Chuang, Shih-Sung

Chuang, Yachen

Chudzinski, Ana Marisa

Chuffa, Luiz Gustavo De Almeida
Chugh, Rishi Man

Chun, Jong Tai

Chun, Kyung-Soo

Chung, Byung Min

Chung, Chaeuk

Chung, Changuk

Chung, Ching-Hu

Chung, Sungjin

Chung, Tae Nyoung

Chutipongtanate, Somchai

Chuva De Sousa Lopes, Susana Marina

Chye, Mee-Len

Chyuan, I-Tsu

Ciaccio, Marcello

Cianciosi, Danila

Ciccarone, Fabio

Cicero, Arrigo

Cichero, Elena

Ciepiela, Olga

Cieslewicz, Artur

Cieślińska, Anna

Cifuentes, Daniel

Cignetti, Alessandro

Cimica, Velasco

Cioce, Mario

Cipolloni, Luigi

Cippitelli, Marco

Cirello, Valentina

Ciribilli, Yari

Cisneros, Bulmaro

Civetta, Alberto

Civita, Prospero

Clarke, Christopher

Clemens, Mark

Clerico, Marinella

Cleveland, Beth M.

Cobley, James

Cobo-Vuilleumier, Nadia

Cocco, Lucio

Coenen, Marieke J.H.

Cohen, Ethan David

Cokkinos, Dennis V.

Colanzi, Antonino

Colasanti, Roberto

Coletti, Dario

Colla, Emanuela

Collart, Martine A.

Collas, Philippe

Collawn, Sherry S.

Colombini, Alessandra

Colosetti, Pascal 
Colunga Biancatelli, Ruben Manuel Luciano Croce, Anna Cleta

Combes, Alex

Combettes, Laurent

Comi, Cristoforo

Comincini, Sergio

Conciatori, Fabiana

Conconi, Maria Teresa

Conde, Javier

Confalonieri, Fabrice

Confalonieri, Marco

Conn, Crystal S.

Conti, Pio

Conversi, David

Coppola, Andrea

Coraux, Christelle

Corazzari, Marco

Corbí, Angel L.

Cordani, Marco

Cordeiro, Yraima

Cordo Russo, Rosalía

Cordo Russo, Rosalía I.

Coria Ortega, Roberto

Cormont, Mireille

Cornelius, Denise

Corradi, Anna

Correia, Sara

Cortese, Barbara

Cortinovis, Cristina

Cossarizza, Andrea

Costa, Alex

Costa, Diana

Costantini, Claudio

Cottrell, Graeme

Coudert, Jerome

Coulon, Stéphane

Coulson, Elizabeth

Courties, Gabriel

Coury, Fabienne

Coutant, Frédéric

Couvineau, Alain

Cowles, Robert

Cox, Laura

Cozar-Castellano, Irene

Craiem, Damian

Cramer, Emily R. A.

Cremisi, Federico

Crescitelli, Rossella

Criado, Gabriel

Crichton, Robert R.

Crinelli, Rita

Crocco, Paolina
Crocè, Lory Saveria

Crocini, Claudia

Crompton, Tessa

Crook, Jeremy M.

Croset, Martine

Crosio, Claudia

Cross, Frederick R.

Cross, Michael

Croteau, Deborah

Crow, Timothy J.

Cuevas-Romero, Estela

Cugliari, Giovanni

Cui, Huachun

Cui, Jianzhou

Cui, Wei

Cui, Weiguo

Cukrowska, Božena

Cullis, Christopher

Culmsee, Carsten

Cusick, John K.

Cvetanovic, Marija

Cyganek, Lukas

Czapiewski, Piotr

Czernicka, Małgorzata

Czosnek, Henryk

Czumaj, Aleksandra

D. Škrbić, Biljana

D'Agostino, Mattia

D'Alessandro, Miriana

D'Amico, Emanuele

Da Costa, Tiago Januário

Da Pozzo, Eleonora

Da Silva, Henrique Borges

Da Silva, Jack

Da Silva, Luis Cláudio Nascimento

Daha, Mohamed

Daher, João P. L.

Dahlin, Joakim

Dai, Dao-Fu

Dai, Zhiyu

Dal Prá, Ilaria

D'Alessandria, Calogero

D'alessio, Alessio

Dalmolin, Rodrigo Juliani Siqueira

D'Alò, Francesco

Damiani, Giovanni

Damiano, Fabrizio

Dandawate, Prasad

D'Andrea, Vito

Dang, Tuyen $\mathrm{T}$. 
Danser, Jan

D'Antongiovanni, Vanessa

Dar, Mohd Saleem

Darabi, Radbod

Darche, Fabrice

Dasanna, Anil K.

Dasgupta, Biplab

Dash, Sabyasachi

Datta, Sandipan

Daub, Steffen

Daubon, Thomas

David Cordonnier, Marie Hélène

David, Laurent I.B.

David, Robert

Davis, Benjamin Michael

Davolos, Domenico

Dawid, Corinna

Day, Andrew S.

Day, Regina M.

Dayem, Ahmed Abdal

De Almeida, Roberto Farina

De Araujo, Heloisa Sobreiro Selistre

De Assis, Leonardo V. M.

De Barrios, Oriol

De Bellis, Luigi

De Benedetti, Stefano

De Bortoli, Marzia

De Brevern, Alexandre G.

De Carvalho, Litia Alves

De Castro, Gabriela

De Ceballos, Maria L.

De Ciuceis, Carolina

De Filippis, Vincenzo

De Francesco, Ernestina Marianna

De Francesco, Francesco

De Francisco, Patricia

De Freitas Fraga, Hugo Pacheco

De Groot, Arjan

De Jonghe, Kris

De Juan Romero, M. Del Camino

De La Rosa, Jorge

De La Serna, Ivana L.

De Lima e Martins Lara, Nathalia

De Lourdes Pereira, Maria

De Luca, Angela

De Luca, Paola

De Maagd, Ruud

De Martin, Sara

De Masi, Luigi

De Noronha, Lucia

De Oliva, Samara Urban
De Pace, Raffaella

De Re, Vallì

De Rivero Vaccari, Juan Pablo

De Rosa, Francesco

De Rossi, Pierre

De Ruijter-Villani, Marta

De Servi, Stefano

De Silva, Pushpamali

De Sire, Alessandro

De Stefani, Diego

De Vitis, Claudia

De Vito, Danila

De Vos, John

De Vries, Martine Charlotte

Dean, Charlotte

Dechat, Thomas

Ded, Lukas

Deftu, Alexandru-Florian

Degano, Irene R.

Deineko, Elena Victorovna

Del Bene, Filippo

Del Fresno Sánchez, Carlos

Del Grosso, Ambra

Del Pozo, Victoria

DeLeon-Pennell, Kristine Y.

Delgado-Vélez, Manuel

Delhanty, Patric

Delitala, Marcello

Della Pepa, Giuseppe

Dell'Amore, Andrea

Delle Monache, Simona

Demiot, Claire

Demuro, Angelo

Demyanenko, Svetlana A.

Denisov, Evgeny

Denolly, Solène

Deppermann, Carsten

Deres, Laszlo

Derler, Isabella

Derouiche, Amin

Derudder, Emmanuel

Des Rieux, Anne

Deschenes, Michael

Detsika, Maria G.

Deutsch, Melanie

Dev, Apurba

Dev, Som

Devers, Emanuel

Devesa, Jesús

DeVito, Nicholas

Devost, Dominic 
Dey, Adwitia

Dey, Kamol

Dey, Nandini

Dhar Dwivedi, Shailendra Kumar

Dhasmana, Anupam

Dhungel, Bijay

Di Bernardo, Giovanni

Di Certo, Maria Grazia

Di Cesare Mannelli, Lorenzo

Di Donato, Marzia

Di Felice, Valentina

Di Fonzo, Alessio

Di Franco, Simone

Di Giacomo, Claudia

Di Giammartino, Dafne Campigli

Di Gioacchino, Mario

Di Girolamo, Nick

Di Meglio, Franca

Di Paola, Rosanna

di Patti Maria Carmela, Bonaccorsi

Di Pietro, Paola

Di Pietro, Roberta

Di Ruscio, Annalisa

Diamanti, Luca

Díaz-Payno, Pedro

Dickinson, Robert

Didangelos, Triantafyllos

Diederich, Sandra

Diederichsen, Louise Pyndt

Dietz, Robert M.

Diez Del Corral, Ruth

Dihazi, Hassan

Dimakopoulos, Yannis

Dimitriadis, George

Dimitroff, Charles J.

Dimopoulos, Meletios-Athanasios

Dinescu, Sorina

Ding, Baojin

Ding, Ling-Wen

Dioguardi, Francesco S.

Diwan, Abhinav

Dix, Richard

Diz-Chaves, Yolanda

Djerada, Zoubir

Djonov, Valentin

Dmitrieva, Renata I.

Dmuchowska, Diana

Do Carmo, Sonia

Do, Minh Truong

Doberentz, Elke

Dobolyi, Arpád
Dobrowolski, Piotr

Dobrzynski, Halina

Doetsch, Paul William

Dohgu, Shinya

Dokudovskaya, Svetlana

Dolivo, David

Dolzani, Paolo

Domenici, Fabio

Domeniconi, Raquel Fantin

Domingos, Pedro $M$.

Dominguez-Vías, German

Domon, Hisanori

Donahue, Seth W.

Donati, Giacomo

Dondossola, Daniele

Doria, Rossella

Dormond, Olivier

D'Oronzo, Stella

Dos Santos, Reinaldo Sousa

Doss, Michael Xavier

Dosset, Magalie

Doulias, Paschalis-Thomas

Doustkhah Heragh, Esmaeil

Dovat, Sinisa

Doye, Valérie

Dragoni, Silvia

Drenjančević, Ines

Dreux, Marlene

Driesen, Ronald B.

Drlica, Karl

Drouet, Emmanuel

Drozd-Sokołowska, Joanna

Drucker, Martin

Drukarch, Benjamin

Drulis-Fajdasz, Dominika

Du, Heng

$\mathrm{Du}$, Shaojun

Du, Xiao-Jun

Du, Ximing

Duarri, Anna

Duarte, Marcio S.

Dubinin, Mikhail

Dubovy, Petr

Dubrova, Yuri

Dubský, Michal

Dubuquoy, Laurent

Ducamp, Sarah

Ducret, Thomas

Dudzińska, Ewa

Duerr, Claudia

Duguez, Stephanie 
Dukhinova, Marina S.

Dumas, Sébastien J.

Dumbauld, Chelsae

Duncan, Greg J.

Duncan, Raymond Scott

Duncan, Steven R.

Dunlop, Elaine

Duntas, Leonidas

Dupont, Geneviève

Durand, Bénédicte

Durek, Thomas

D'Uscio, Livius V.

Dutt, Taru S.

Dutta, Pranabananda

Dutta, Suman

Duvigneau, J. Catharina

Dux, Mária

Dvorak, Zdenek

Dye, Danielle E.

Dyer, Arthur

Dymova, Maya

Dziedziech, Alexis

Dziegiel, Piotr

Dziewięcka, Marta

Ear, Jason

Eaton, Douglas

Ebeed, Heba

Ebstein, Frédéric

Eckhardt, Matthias

Eckhart, Leopold

Eder, Iris E.

Eeckhoute, Jérôme

Efrat, Shimon

Eftekharpour, Eftekhar

Egan, Colin Gerard

Ehinger, Johannes K.

Ehlting, Christian

Ehrlich, Aliza Toby

Eid, Nabil

Eide, David

Eiró, Noemí

Eisel, Ulrich L. M.

Eixarch, Herena

Ejaz, Asim

El Alaoui, Hicham

El-Aarag, Bishoy Y. A.

Elchaninov, Andrey

Eldridge, Sandy

Eleftheriou, Eleftherios P.

Elena, Adinolfi

El-Esawi, Mohamed A.
El-Far, Ali H.

Eliseeva, Irina

Eljaafari, Assia

Eljaszewicz, Andrzej

ElKamhaw, Ahmed

El-Khamisy, Sherif

Ellepola, Kassapa

Ellis, Ronald

El-Mochtar, Choaa

Elmonem, Mohamed

Elroy-Stein, Orna

Elsayed, Mansour

Elsherbiny, Nehal

El-Shishtawy, Mamdouh

Elvire, Pons-Tostivint

Emery, R. J. Neil

Emmerson, Elaine

Emons, Günter

Engeland, Kurt

Engström, Ylva

Enguita, Francisco J.

Eoli, Marica

Epp, Jonathan

Eppard, Elisabeth

Erickson, David

Ericson, Marna E.

Erjavec, Gordana Nedic

Escolà-Gil, Joan Carles

Escors, David

Escribese, María M.

Eskelinen, Eeva-Liisa

Esmaili, Mansoore

Esnaut, Stephane

Espada, Jesús

Espana, Edgar

Espel, Enric

Espíndola, Otávio

Espinosa, Lluis

Esposito, Giuseppe

Esser, Nathalie

Esteban, María

Esteban, Raquel

Esteban, Vanesa

Esteves, Ana Raquel

Estus, Steven

Eto, Yoshikastu

Eufemi, Margherita

Eugenin, Eliseo

Eun, Hyuk Soo

Éva, Csaba

Éva, Mikó 
Evani, Shankar Jaikishan

F. G. Dias, Fernanda

Fabczak, Hanna

Fabi, João Paulo

Fabian, Zsolt

Fabiana Fernandes, Bressan

Facchiano, Antonio

Facchin, Federica

Faciotti, Federica

Fafián-Labora, Juan A.

Faget, Julien

Fagone, Paolo

Fahad, Shah

Fahrner, Marc

Failla, Cristina M.

Fais, Stefano

Faissner, Andreas

Fajkus, Jiří

Falasca, Laura

Falkmer, Ursula Gerda Inge

Fall, Mamadou

Fang, Evandro Fei

Fang, Qingming

Fang, Su-Chiung

Fang, Yu-Wei

Fantini, Damiano

Farcasanu, Ileana C.

Faris, Pawan Sirwan

Farkaš, Vladimír

Farmer, Diana

Farnsworth, Nikki L.

Faron-Górecka, Agata

Farooqi, Ammad Ahmad

Fasciana, Teresa

Fasolato, Cristina

Fatkhudinov, Timur Kh.

Faustini, Gaia

Favaloro, Emmanuel J.

Favi, Evaldo

Faviana, Pinuccia

Favier, Julien

Fehrenbach, Daniel

Feith, David

Fejtova, Anna

Fekete, Tünde

Felcht, Moritz

Feldo, Marcin

Feliciani, Claudio

Félix, Luís M.

Feltham, Rebecca

Fendt, Markus
Feng, Bingjian

Feng, Sheng-Wei

Feola, Andrew

Fereira Garrudo, Fábio Filipe

Ferguson, Stephen

Ferlazzo, Nadia

Fernandes, Hugo

Fernández Galilea, Marta

Fernández Ponce, Cecilia Matilde

Fernandez, Eduardo

Fernandez, Marina O.

Fernández, Sara Rosalía Morcuende

Fernández, Vanesa Martín

Fernández-Albarral, José A.

Fernández-Carrión, Rebeca

Fernández-Martínez, Eduardo

Fernández-Sánchez, Laura

Fernández-Solà, Joaquim

Fernandez-Twinn, Denise

Ferree, Patrick

Ferreira Cerca, Sebastien

Ferreira, Fernando

Ferreira, Jorge

Ferreira, Rodrigo

Ferreira, Viviana

Ferreira-da-Silva, Francisco

Ferrucci, Michela

Fessler, Johannes

Fesus, Laszlo

Fiedorowicz, Ewa

Figueiredo, Bonald

Filatov, Alexander

Filek, Maria

Filep, János G.

Filigheddu, Nicoletta

Filipek, Agnieszka

Filipek, Anna

Filipič, Bratko

Filipovic, Natalija

Filippou, Panagiota S.

Filosa, Jessica

Findlay, Geoffrey D.

Finelli, Carmine

Finkenzeller, Günter

Finocchiaro, Gaetano

Finsterer, Josef

Fior, Rita

Fiorani, Mara

Fiorotto, Romina

Firestein, Bonnie L.

Firestein, Ron 
Fischer, Andreas

Fischer, Michael B.

Fischer, Ulrike

Fisher, Heidi

Fiszer, Agnieszka

Fitzhugh, Kirk

Flagg, Thomas

Fleming, Alastair

Fleming, Cassandra

Fletcher, Nicola F.

Flockerzi, Veit

Florán, Benjamín

Florean, Cristina

Flores, Ana

Fogacci, Federica

Folco, Giancarlo

Follenzi, Antonia

Fonseca-Alves, Carlos Eduardo

Forini, Francesca S.

Forleo, Giovanni B.

Forma, Alicja

Fornaguera, Cristina

Fornasaro, Stefano

Forni, Monica

Foroncewicz, Bartosz

Forrest, Douglas

Forstmeier, Wolfgang

Fort, Philippe

Forte, Maurizio

Fortschegger, Klaus

Fortunel, Nicolas

Foschino-Barbaro, Maria Pia

Fossarello, Maurizio

Fossati, Silvia

Fowkes, Rob

Fragoso, Rita

Fraineau, Sylvain

Francavilla, Chiara

Francés, Rubén

Francis, Heather

Franco, Antonietta

Franco, Omar

Franco, Pierfrancesco

Franco, Santos

Francs-Small, Catherine Colas Des

Frank, Samuel

Frankel, Adam

Fransen, Marieke F.

Franzin, Rossana

Frasca, Loredana

Fraschini, Roberta
Frassanito, Antonella

Frati, Alessandro

Freen-van Heeren, Julian

Freitas, Vanessa Morais

Freude, Kristine

Friant, Sylvie

Frick, Luciana

Fridrichova, Ivana

Friis-Hansen, Lennart

Frisoni, Tommaso

Frontiñán-Rubio, Javier

Frontini, Andrea

$\mathrm{Fu}$, Mingui

Fuchs, Marc

Fuentealba, Jorge

Fujii, Hideki

Fujii, Kazuyasu

Fujii, Masayuki

Fujimura, Atsushi

Fujishima, Hiroshi

Fujita, Akikazu

Fujitani, Masashi

Fukagawa, Tatsuo

Fukasawa, Takemichi

Fukazawa, Takuya

Fukushima, Atsushi

Fulci, Valerio

Fulgenzi, Gianluca

Funaki, Makoto

Fuoco, Claudia

Furuse, Junji

Fusar-Poli, Laura

Fuster, Daniel

Futrega, Kathryn

Gabrielli, Armando

Gabrielli, Brian

Gabryelska, Marta

Gad, Ahmed

Gad, Annica K. B.

Gaddam, Ravinder Reddy

Gaffke, Lidia

Gage, Matthew

Gai, Marta

Gaidano, Gianluca

Gaillard, Afsaneh

Gaillard, Hélène

Gaillochet, Christophe

Galbiati, Mariarita

Galbiati, Silvia

Galderisi, Umberto

Galešić, Krešimir 
Galietta, Luis

Galigniana, Mario D.

Gallardo, M. Esther

Gallardo, Nilda

Gallego, Monica

Gallego, Óscar S.

Gallo, Gaetano

Gallorini, Marialucia

Galoian, Karina

Galuska, Sebastian P.

Gaman, Mihnea-Alexandru

Gamarra, Lionel

Gambaryan, Stepan

Gamboa-deBuen, Alicia

Gamez, Stephanie

Gamsjaeger, Sonja

Gandasi, Nikhil R.

Gandhi, Jatin Sundersham

Gangadaran, Prakash

Gantenbein, Benjamin

Gao, Gary

Gao, Peisong

Gao, Shouguo

Gao, Shuai

Gaponenko, Vadim

Garab, Győző

Garagiola, Umberto

Garagna, Silvia

Garban, Frédéric

Garcia Alvarez, Olga

García Gonzalo, Francesc

Garcia Gutierrez, Lucia

García Meilán, Irene

García Souto, Daniel

García, Gabriela

Garcia, Victor

Garcia-Alvarez, Ana

García-Ayllón, María-Salud

García-Barrado, María José

Garcia-Gil, Mercedes

García-González, Víctor

García-Guerrero, Estefanía

Garcia-Macia, Marina

García-Murria, María J.

García-Plazaola, José Ignacio

García-Ríos, Estéfani

García-Silva, Susana

Gardikis, Konstantinos

Gardoni, Fabrizio

Garg, Manoj

Garmash, Elena V.
Garofalo, Stefano

Garozzo, Domenico

Garstka, Malgorzata Anna

Garten, Antje

Garvin, David

Gasbarrini, Alessandro

Gasiūnas, Giedrius

Gasparini, Clelia

Gasperi, Valeria

Gassaway, Brandon Mark

Gaßler, Nikolaus

Gastaminza, Gabriel

Gaston, J. S. Hill

Gates, Colin

Gatzoulis, Konstantinos A.

Gaudio, Eugenio

Gauthier, Benoit

Gautier, Mathieu

Gavaldà-Navarro, Aleix

Gee, Christine E.

Gęgotek, Agnieszka

Gehrke, Thomas

Geisinger, Adriana

Geismann, Claudia

Genc, Sermin

Genders, Amanda

Genikhovich, Grigory

Gentile, Alessandra

Gentile, Antonietta

Gentile, Fabrizio

Gentile, Vittorio

Geoffroy, Cédric G.

Georgakilas, Alexandros

George, Rajani

Georges, Steven

Georgescu, Adriana

Gerard, Ludovic

Gerasimenko, Oleg

Gerbal-Chaloin, Sabine

Gerbeau-Pissot, Patricia

Gerhold, Kerstin

Geri, Pietro

Gerlich, Wolfram

Germana, Antonino

Gerrard, Gareth

Gersch, Malte

Gershon, Michael

Gertsch, Jürg

Gessi, Stefania

Geuna, Massimo

Geva-Zatorsky, Naama 
Ghanim, Bahil

Ghasemi, Mehdi

Ghasghaei, Troy

Gherardi, Gaia

Ghezzi, Daniele

Ghiasi, Seyed Mojtaba

Ghimire, Kedar

Gholamreza, Gohari

Giacomini, Arianna

Gianesello, Lisa

Giangrande, Angela

Giangrieco, Ivana

Giannoccaro, Maria Pia

Gibeaux, Romain

Gibellini, Lara

Giblin, Frank

Gibson, Spencer

Gica, Nicolae

Gieras, Anna

Gigant, Benoît

Gigante, Antonietta

Gijbels, Eva

Gil Fernández, Vanessa

Gil, Cristiane Damas

Gil, Ziv

Gillespie, Peter G.

Gillet, Reynald

Gimble, Jeffrey

Gioia, Magda

Giovambattista, Andrés

Giovarelli, Matteo

Giovarelli, Mirella

Giraud, Marie Noëlle

Girault, Alban

Gires, Olivier

Giri, Anil K.

Girolamo, Francesco

Girstun, Agnieszka

Gissen, Paul

Giuffrida, Rosario

Giurisato, Emanuele

Giuseppina, Basini

Gkogkas, Christos

Gkretsi, Vasiliki

Gladish, Daniel K.

Glaser, Karin

Glover, David

Gnanapragassam, Vinayaga Srinivasan

Gnanasundram, Sivakumar Vadivel

Gobeil, Fernand, Jr.

Goda, Nobuhito
Goel, Peeyush N.

Goh, Sung-Ho

Gokhale, Nandan S.

Goldstein, Allan

Gołembiowska, Krystyna

Golenhofen, Nikola

Golia, Evangelia

Golovics, Petra Anna

Gomarasca, Marta

Gomes, Ana Rita

Gomes, Eduardo D.

Gomes, Fabio

Gomes, Joao R.

Gómez Orte, Eva María

Gomez, Nidia

Gómez-Torres, Maria José

Gomzikova, Marina

Gonçalves, Ana

Gonçalves, Céline S.

Gonfiotti, Alessandro

Gonkowski, Sławomir

Gonzalez Espinosa, Claudia

Gonzalez Torralva, Fidel

Gonzalez, Antonio

Gonzalez, Francisco Garcia

Gonzalez, Victor

González-Arenas, Aliesha

González-Espinosa, Claudia

González-Fernández, Carlos

Gonzalez-Menendez, Pedro

González-Mesa, Ernesto

González-Navarro, Herminia

Gonzalez-Pujana, Ainhoa

González-Reyes, Acaimo

Gopal, Keshav

Gorbacheva, Liubov

Gorbatyuk, Oleg S.

Gorbe, Aniko

Gorchakov, Andrey A.

Gorgulu, Kivanc

Gorio, Alfredo

Górna, Maria W.

Gorrie, Catherine Anne

Gorvel, Laurent

Goswami, Mukunda

Goto, Yuhei

Gotta, Monica

Götz, Klaus-Peter

Goudarzi, Salman

Gould, Robert

Gozal, Evelyne 
Grabarek, Beniamin Oskar

Grabowska, Iwona

Gracia Lostao, Ana Isabel

Grade, Sofia

Graef, Martin

Gräf, Ralph

Gragnano, Felice

Graham, Annette

Graille, Marc

Gramignoli, Roberto

Gramolelli, Silvia

Granados-Principal, Sergio

Granados-Principal, Sergio M.

Granito, Alessandro

Granot, Zvika

Grãos, Mário

Grassi, Guido

Grau, James W.

Graumann, Peter

Gray, Douglas A.

Graziella, Messina

Greber, Urs

Greene, Nicholas P.

Greenhalgh, David

Grefhorst, Aldo

Gregor, Martin

Gregory, Christopher D.

Greiner, Johannes

Grela, Przemysław

Greotti, Elisa

Grespan, Eleonora

Grewal, Thomas

Grieve, David

Grigorov, Ilijana

Grigoryan, Eleonora N.

Grillo, Elisabetta

Grimm, Marcus

Grimm, W. D.

Gristina, Roberto

Grobe, Kay

Gröbner, Gerhard

Grocholewicz, Katarzyna

Groschner, Klaus

Grossman, Lawrence I.

Grote, Karsten

Grove, Joe

Groveman, Bradley

Gruba, Natalia

Grusch, Michael

Grützkau, Andreas

Gryder, Berkley
Grzela, Katarzyna

Grzmil, Paweł

Grzymajlo, Krzysztof

Grzywa, Tomasz

$\mathrm{Gu}$, Siyi

$\mathrm{Gu}$, Wei

Gu, Zhenglin

Gualberto, Jose

Guang, Shouhong

Guatimosim, Silvia

Gude, Natalie A.

Gudermann, T.

Gueron, Geraldine

Guerra, Flora

Guerrero, Adan

Guerrero, Sandra M. Martin

Guescini, Michele

Guevara Garcia, Angel Arturo

Guevara González, Ramón

Guha, Prasun

Guihot, Amélie

Guillamat-Prats, Raquel

Guillén Viejo, Carlos

Guillén, Carlos Viejo

Guillen-Grima, Francisco

Guilpain, Philippe

Guimarães, Fernando

Guimarães, Simone E. F.

Guinamard, Romain

Guiot, Caterina

Guix, Francesc Xavier

Güldiken, Nurdan

Gulino, Rosario

Gulluni, Federico

Gulya, K.

Gulyaeva, Natalia V.

Gumerov, Vadim M.

Guo, Lilong

Guo, Qingbin

Guo, Yong

Gupta, Anisha

Gupta, Kuldeep

Gurevich, Vsevolod V.

Gurskaya, Nadya G

Gurzov, Esteban N.

Gutiérrez, Laura

Gutiérrez-Cañas, Irene

Gutierrez-Juarez, Roger

Gutmann, Marcus

Guttery, David S.

Guzhova, Irina V. 
Gyoergy, Bence

Hadjipavlou-Litina, Dimitra

Hagedorn, Monica

Hahne, Jens

Haigh, Anthony

Hajduch, Eric

Hakovirta, Harri H.

Hale, Taben M.

Halle, Stephan

Haller, Thomas

Halliwell, Robert F.

Halloran, Kieran

Hálová, Ivana

Ham, Daniel

Hamad, Mawieh A.

Hamaguchi, Tsuyoshi

Hamaratoğlu, Fisun

Hamazaki, Jun

Hamieh, Mohamad

Hamilton, Russell

Hammer, Sandra Beer

Hammers, Christoph M.

Hamon, Morgan

Hamperl, Stephan

Han, Inbo

Han, Jaeseok

Han, Pingping

Han, Renzhi

Handa, Avtar K.

Hanga, Mariana Petronela (Petra)

Hannan, Katherine M.

Hanschen, Erik R.

Happel, Christine

Haraguchi, Tokuko

Hardien, David

Hardtke-Wolenski, Matthias

Hardy, Rowan S.

Harel, Amnon

Hargreaves, Iain

Hargreaves, Iain Parry

Harijith, Anantha

Härkönen, Pirkko

Harlé, Alexandre

Harper, Matthew T.

Harris, David T.

Harris, Norman R.

Harris, Raymond

Harrison, Patrick

Hartley, Oliver

Hartman, Mariusz

Hartmann-Petersen, Rasmus
Haruna, Takenori

Hascall, Vincent

Hasegawa, Natsuki

Hashimoto, Makoto

Hasipek, Metis

Hassan, Sherif T. S.

Hatem, Stéphane N.

Hatori, Yuta

Hatzimichael, Eleftheria

Hatzoglou, Maria

Hau, Peter

Hau, Raymond K.

Haugsten, Ellen

Hauptmann, Steffen

Hautz, Theresa

Hawinkels, Lukas

Hayashida, Kenji

Haybaeck, Johannes

He, Baoye

He, Junmin

He, Liangcan

He, Ling

He, Xiaochen

He, Yaowu

Heath, Paul

Hebeda, Cristina B.

Hecker, Andreas

Hedman, Klaus

Hedwig, Hedwig Sutterlüty-Fall

Hegde, Vijay

Hegermann, Jan

Heidor, Renato

Heidstra, Renze

Heijman, Jordi

Heinisch, Jürgen

Heinrich, Bernd

Heinz, William F.

Hejmej, Anna

Helgason, Vignir

Hellemons, Merel E.

Heller, Janosch

Hellmen, Eva

Hellweg, Christine

Helm, Alexander

Helmbacher, Françoise

Hendrikx, Marc

Hendrix, Mary

Heng, Tracy

Hengst, Ludger

Hengstler, Jan

Hénique, Carole 
Henrich, Dirk

Henriksson, Johan

Henriques-Pons, Andrea

Henry, Linda L.

Henry, Ryan A.

Henson, Jeremy

Herbort, Carl P.

Hermouet, Sylvie

Hernández, M. Luisa

Herndler-Brandstetter, Dietmar

Herr, Ingrid

Herrbach, Etienne

Herrera, Antonio J.

Herrero Ezquerro, María Trinidad

Hervera, Arnau

Heß, Jochen

Hessheimer, Amelia J.

Hester, Joanna

Hetman, Michal

Heumann, Rolf

Hidalgo, Juan

Hilfiker, Sabine

Hilhorst, Marc

Hill, April L.

Hill, Reginald

Himanshu, Arora

Hime, Gary

Himoto, Takashi

Hinds, Terry D.

Hippler, Michael

Hirakawa, Yosuke

Hirano, Katsuya

Hirata-Tsuchiya, Shizu

Hiromatsu, Yuji

Hirsch, Emilio

Hitomi, Kiyotaka

Hizi, Amnon

Ho, Gwo-Tzer

Hochheiser, Katharina

Hochrainer, Karin

Hofer, Edith

Hofer, Matthias D.

Hoffer, Barry

Hoffmann, Uwe

Hogue, Brenda G.

Hohenfellner, Katharina

Hohmann, Tim

Hojan, Katarzyna

Hollmén, Maija

Hollywood, Jennifer A.

Holubova, Monika
Holzer, Max

Hombauer, Hans

Homma, Takujiro

Honaramooz, Ali

Hong, Chien-Tai

Hong, Jiann-Ruey

Hong, Seok-Ho

Hong, Xupeng

Hoppler, Stefan

Hori, Toshiyuki

Horsfall, Laura

Horsfield, Julia A.

Hortobagyi, Tibor

Hoshiba, Takashi

Hoshino, Fumi

Hosui, Atsushi

Ho-Tin-Noé, Benoît

Hottiger, Michael

Hotto, Amber M.

Hough, Kenneth

Houhamdi, Linda

House, Imran

Houtman, Jon

Hovatta, Iiris

Hoyer, Juliane

Hoyer-Fender, Sigrid

Hritcu, Lucian

Hsiao, Chengchih

Hsiao, Hui-Hua

Hsieh, Ming-Ju

Hsin, I-Lun

Hsu, Li-Chi

Hsu, Li-Sung

Hsu, Ren-Jun

Huang, Cao

Huang, Chiung-Kuei

Huang, $\mathrm{Hu}$

Huang, Huocong

Huang, Jing

Huang, Lei

Huang, Mingchao

Huang, Shar Yin Naomi

Huang, Shuanglong

Huang, Shu-Pin

Huang, Zixia

Huber, Eva M.

Huber, Michael

Huber, Rene

Huber, Samuel

Hudson, Natalie

Hueso, Miguel 
Hughes, Timothy

Huh, Jin Hoe

Hukowska-Szematowicz, Beata

Humphries, Brock

Hung, Chung-Lieh

Hung, Shih-Ya

Huo, Yuqing

Huot, Joshua R.

Hur, Jae Young

Hurley, James B.

Husa, Ana-Maria

Husnjak, Koraljka

Hussain, Ali A.

Hwang, Heeyoun

Hwang, In Koo

Hwang, Inhwan

Hytti, Maria

Iaccarino, Ciro

Iaccino, Enrico

Iacoangeli, Alfredo

Iakimova, Elena T.

Iannelli, Domenico

Ibrahim, Sulaiman S.

Ichii, Hirohito

Ientile, Riccardo

Ievinsh, Gederst

Ievinsh, Gederts

Iguchi, Yohei

Iijima, Noriaki

Ijima, Hiroyuki

Ikeda, Masahiro

Ikegami, Tadashi

Ilchibaeva, Tatiana

Ille, Fabian

Imai, Yasuo

Imamura, Masahiro

Imbach, Paul A.

Imbert, Véronique

Immenschuh, Stephan

Imperio, Daniela

Ines, Luis

Infante, Arantza

Inga, Alberto

Ingley, Evan

Iniesta Cuerda, María

Inoue, Takafumi

Inyushin, Mikhail Y.

Iommelli, Francesca

Iop, Laura

Iorio, Egidio

Iqbal, Jahangir
Irfan, Mohammad

Irini, Evnouchidou

Isaeva, Marina P.

Ishigaki, Yasuhito

Ishihara, Katsuhiko

Ishikawa, Kiyotake

Ishikawa, Yasuyuki

Ishino, Yoshizumi

Iskender, Ilker

Islas Osuna, Maria Auxiliadora

Isupov, Michail

Itatani, Yoshiro

Ito, Genta

Ito, Hisashi

Ivan Jose, Vechetti

Ivanova, Margarita

Ivković, Vanja

Iwaszkiewicz-Grzes, Dorota

Iyer, Aishwarya

Iyoda, Masayuki

Izawa, Takashi

Izdebska, Magdalena

Jablonska, Ewa

Jabłońska, Jadwiga

Jackowiak, Hanna

Jackson, Joshua G.

Jackson-Weaver, Olan

Jacob, Fadi

Jacobo-Herrera, Nadia Judith

Jacobsen, Elizabeth

Jacquelot, Nicolas

Jacquemet, Guillaume

Jacquot, Yves

Jadiya, Pooja

Jafari, Abbas

Jafri, Mohsin

Jaganjac, Morana

Jaime, Diego Franco

Jain, Manish

Jakimowicz, Dagmara

Jakovlevic, Dragana

Jalkanen, Sirpa

James, Joel

James, Richard G.

Jamroz-Wisniewska, Anna

Jancura, Daniel

Janda, Elzbieta

Jang, Hyosun

Jang, KyuYun

Jang, Sung-Wuk

Jankowska, Elzbieta 
Jansen, Christian

Jansen, Diahann

Jansen, Gert

Jansson, Deidre

Jaraiz, Myriam

Jarome, Timothy

Jarret, Robert

Jarząb, Barbara

Jasek, Monika

Jastrzebska, Beata

Javaheri, Tahereh

Javier Jiménez-Jiménez, Felix

Javier, Raya-González,

Jayawardena, Thilina U.

Jazirehi, Ali R.

Jégo, Gaëtan

Jekabsone, Aiste

Jeltsch, Michael

Jeong, Goo-Bo

Jeong, Se Kyoo

Jeschke, Udo

Jeseta, Michal

Jeyaseelan, Samithamby

Jezierski, Anna

Jha, Narendra

Jhanji, Vishal

Jia, Baolei

Jiang, Jhih-Hang

Jiang, Li

Jiang, Peihua

Jimbo, Mitsuru

Jiménez-González, Gema

Jiménez-Mateos, Eva María

Jochum, Christoph

Joffre, Carine

Johansson, Anders

Johnson, Dan

Johnson, Luise

Johnstone, Cameron

Johnstone, Edward

Johnstone, Elizabeth

Jolicoeur, Mario

Joly, Jean Stéphane

Jones, Marjorie

Jorge, Erika

Jorrin-Novo, Jesus V.

Joshi, Amit

Joshi, Pushpa Raj

Joshi-Barve, Swati

Joubert, Annie

Joubert, Bastien
Jouhet, Juliette

Jovanov-Milošević, Nataša

Juan, Manel

Juarranz, Yasmina

Juiz-Valiña, Paula

Jullienne, Amandine

Julovi, Sohel M.

Jung, Cho Rok

Junion, Guillaume

Junker, Anna

Juranek, Judyta Karolina

Jurášek, Michal

Jurič, Damijana Mojca

Jurisic, Vladimir

Jurk, Kerstin

Jurkowska, Renata Zofia

Kabir, Mohammad Faujul

Kachamakova-Trojanowska, Neli

Kaether, Christoph

Kaewput, Wisit

Kahraman Dirice, Sevim

Kaji, Hiroshi

Kalamajski, Sebastian

Kaleta, Beata

Kalirai, Helen

Kalmann, Rachel

Kaltenecker, Doris

Kaltschmidt, Christian

Kamara, Mohamed

Kamen, Amine

Kamihira, Masamichi

Kaminska, Bozena

Kanai, Yuta

Kanamarlapudi, Venkateswarlu

Kanaujiya, Jitendra

Kanda, Tatsuo

Kandasamy, Palanivel

Kandasamy, Richard Kumaran

Kandeel, Mahmoud

Kaneda, Shohei

Kang, Eunju

Kang, Jeehoon

Kang, Jianming

Kang, Ju-Hee

Kang, Kyung Pyo

Kang, Young-Hee

Kano, Kiyoshi

Kapetanaki, Maria

Kapus, András

Kapustin, Alexander

Karagiannis, Evangelos 
Karakesisoglou, Iakowos

Karakostis, Konstantinos

Karalija, Erna

Karamichos, Dimitrios

Karanika, Styliani

Karasawa, Ken

Karasuyama, Hajime

Karbowniczek, Joanna

Kardassis, Dimitris

Kardos, Julianna

Karimi, Khalil

Karisola, Piia

Karki, Pratap

Karlin, Eric F.

Karr, Timothy

Karsak, Meliha

Karthik, Mallikaraman

Karwowski, Boleslaw

Kasatkina, Ludmila A.

Kaschina, Elena

Kashanchi, Fatah

Kasimatis, Katja R.

Kasirer-Friede, Ana

Kasparov, Sergey

Kasprzak, Aldona

Kassan, Modar

Kassis, Ibrahim

Kasuga, Kensaku

Kataoka, Naoyuki

Kataoka, Tatsuki R.

Kathare, Praveen Kumar

Kato, Takafumi

Katsinelos, Taxiarchis

Katzin, Alejandro M.

Kaufman, Dan

Kaulich, Manuel

Kaur, Gagandeep

Kaushik, Garima

Kavanagh, Dean

Kawabata, Atsufumi

Kawabata, Shinji

Kawaguchi, Nanako

Kawai, Tatsuo

Kawakami, Toshi

Kazui, Toshinobu

Kazunori, Tomita

Ke, Liang-Yin

Kekalainen, Eliisa

Keklikoglou, Ioanna

Keller, Jonathan R.

Kemmerling, Ulrike
Kempaiah, Prakasha

Kempisty, Bartosz

Kendall, Tim

Kerdine-Römer, Saadia

Keresztes, Attila

Kerr, Nadine A.

Keshari, Sunita

Ketteler, Robin

Khairnar, Vishal S.

Khalil, Ahmed Mohamed Ali

Khan, Ilyas

Khan, Sabbir

Khan, Zeeshan Ahmad

Khanam, Arshi

Khaperskyy, Denys A.

Kharaghani, Davood

Khasabov, Sergey G.

Khatami, Mahin

Kherraf, Zine-Eddine

Khiati, Salim

Khodzhaeva, Zul'Fiya Sagdullaevna

Kholová, Ivana

Khoo, Kay-Hooi

Khosla, Aashima

Kiang, Juliann G.

Kiecker, Clemens

Kiela, Pawel

Kiesel, Barbara

Kil, Eui-Joon

Killinger, Bryan Andrew

Kim, Alfred

Kim, Hahn Young

Kim, Hei-sung

Kim, Ho Yeon

Kim, Ho-Seong

Kim, Hyun Koo

Kim, Hyung-Sik

Kim, Hyun-Jung

Kim, In-Jung

Kim, Jaebong

Kim, Jaesang

Kim, Jaesung

Kim, Jaeyoon

Kim, Jong-Eun

Kim, Jong-Hyuk

Kim, Ki-Young

Kim, Kyeong-Man

Kim, Kyoungtae

Kim, Kyung-Min

Kim, Min Jung

Kim, Min-Soo 
Kim, Nam Deuk

Kim, Sahn-Ho

Kim, Sang Woo

Kim, Sang-Nam

Kim, Seongjae

Kim, Shin

Kim, Sun-young

Kim, Tae-Don

Kim, Woojin

Kim, Yoosik

Kim, Yu-Jin

Kimura, Kiminori

Kimura, Nobuyuki

Kino, Tabito

Kinose, Yasuto

Kinoshita, Hiroyuki

Kins, Stefan

Király, Lóránt

Kirches, Elmar

Kireev, Igor I.

Kirkland, Jacob G.

Kishi, Yusuke

Kishimoto, Jiro

Kiss, Andrea

Kiss, Anna L.

Kiss, Csongor

Kiss, Lorand

Kiss, Rita

Kiss, Robert

Kissa, Karima

Kitagawa, Seiichi

Kitamura, Daisuke

Kitamura, Kenji

Kitano, Takeshi

Kitchen, Philip

Kitowska, Kamila

Kitt, Jay P.

Kittel, Agnes

Klaile, Esther

Klar, Agnes

Klavins, Linards

Kleeff, Jörg

Klein, Stefanie

Kleinman, Hynda

Klementieva, Oxana

Klempnauer, Karl Heinz

Kleuser, Burkhard

Klewicka, Elzbieta

Klimaszewski, Lars

Klimczak, Aleksandra

Klimek-Ochab, Magdalena
Kloc, Malgorzata

Klymiuk, Michele

Knapczyk-Stwora, Katarzyna

Knezevic, Jelena

Knight, Jim

Knuplez, Eva

Kobarg, Jörg

Kobayashi, Kenta

Kobayashi, Koichi

Kobayashi, Nobumichi

Kobayashi, Satoru

Kobayashi, Tatsuya

Kobayashi, Yoshiro

Kobeissy, Firas

Kobierzycki, Christopher

Koch, Karl-Wilhelm

Kochneva, Galina

Kočí, Zuzana

Koduru, Srinivas V.

Koeleman, Bobby

Koestenberger, Martin

Kohorn, Bruce

Koinis, Filippos

Koivisto, Ari

Kojima, Kenji

Kok, Chung Hoow

Kolackova, Ivana

Kolaczkowska, Elzbieta

Koldehoff, Michael

Kolosova, Nataliya

Kołton, Anna

Komleva, Yulia K.

Kondo, Tomo

Koninckx, Philippe R.

Konjar, Špela

Konjevoda, Paško

Konstantinidis, Theocharis G.

Kontaraki, Joanna E.

Konturek, Peter Christopher

Koos, Björn

Kopjar, Mirela

Kopp, Benjamin T.

Korać, Petra

Korczeniewska, Olga

Kordek, Agnieszka

Kordowitzki, Paweł

Kordyum, Elizabeth L.

Korfitis, Chrysovalantis

Korkmaz, Yüksel

Korkotian, Eduard

Korolenko, Tatiana A. 
Korolkova, Olga Y.

Korosoglou, Grigorios

Korthagen, Nicoline M.

Kortholt, Arjan

Korzhevskii, Dmitrii

Kosan, Christian

Koscielny, Sven

Kosowska, Agnieszka

Kostallari, Enis

Kostas, Michal

Kostić, Marina

Kostyuchenko, Roman

Kosuge, Yasuhiro

Kot, Karolina

Kothari, Vishal M.

Kottilil, Shyam

Kottmann, Andreas H. H.

Kounatidis, Ilias

Kovac, Stjepana

Kováčik, Andrej

Kovács, Árpád Ferenc

Kovács, Krisztina

Kovács-Öller, Tamás

Kovalszky, Ilona

Kowalewska, Łucja

Kowalska, Maria Anna

Kozhevnikova, Oyuna

Kozieł, Edmund

Kozieł, Monika

Koziorowski, Dariusz

Kozlova, Elena

Kozlowska, Emilia

Krag, Thomas O.

Krapf, Dario

Krasikova, Alla

Krasikova, Yuliya S.

Kratz, Ewa Maria

Krause, Matthias

Krawiec, Paulina

Kreis, Nina-Naomi

Krick, Stefanie

Krishna, Gokul

Krishna, Suresh Babu Naidu

Krishnakumar, Devadas

Krishnamoorthy, Raghu R.

Krishnasamy, Gopinath

Kristen W., Cohen

Kristian, Tibor

Krohne, Georg

Kron, Ivan
Kropp, Martina

Krskova, Lenka

Krstic, Jelena

Krueger, Andreas

Krüger, Marcus

Kruger, Marlena

Kruglov, Andrey

Krzystek-Korpacka, Malgorzata

$\mathrm{Ku}$, Wei-Chi

Kuang, Zheng

Kubala, Lukáš

Kubota, Hiroshi

Kucharska-Mazur, Jolanta

Kucharz, Eugene

Kuehnel, Florian

Kujan, Omar

Kuliczkowski, Kazimierz

Kulik, Anna

Kulikov, Alexander V.

Kulkarni, Dhananjaya

Kulkarni, Priyanka

Kullberg, Susanna

Kulus, Dariusz

Kumar, Ajay

Kumar, Anoop

Kumar, Anuj

Kumar, Ashok

Kumar, Kishore

Kumar, Manu

Kumar, Mukesh

Kumar, Thallapuranam Krishnaswamy

Suresh

Kumari, Daman

Kumari, Meena

Kumari, Sonam

Kümmerer, Beate M.

Kundariya, Hardik

Kundu, Kiran

Kunkel, Thomas

Kunz, Wolfram

Kunze, Markus

Kuo, Hsiaohsuan

Kura, Branislav

Kurakula, Kondababu

Kurczyńska, Ewa

Kurimoto, Takuji

Kuroda, Hiroaki

Kurokawa, Manabu

Kurose, Hitoshi

Kurtz, Andreas

Kuryk, Lukasz 
Kuryłowicz, Alina

Kushima, Miki

Kuter, Katarzyna

Kuthati, Yaswanth

Kutikhin, Anton

Kuzel, Timothy M.

Kuźnik-Trocha, Kornelia

Kvandova, Miroslava

Kwakkel-van Erp, Hanneke

Kwiatkowska, Katarzyna

Kwok, Hoi-Hin

Kwon, Byungsuk

Kwon, Hyuk-Joon

Kwon, Oh Sung

Kwon, So Hee

Kyriakoulis, Konstantinos G.

L. Balint, Balint

La Porta, Caterina

La Rosa, Piergiorgio

Laboisse, Christian L.

Labouta, Hagar Ibrahim

Labrador, Jorge

Lacal, Juan Carlos

Lach, Michał Stefan

Lacko, Andras

Lacobescu, Gabriela-Eugenia

Lacroix, Benjamin

Laczmanski, Lukasz

Ladani, Amit

Ladekarl, Morten

Ladilov, Yury

Ladomersky, Erik

Lagali, Pamela

Lahlil, Rachid

Lahmer, Tobias

Lai, Wing-Fu

Lai, YingJu

Lallemand, Francois

Lalli, Enzo

Lalonde, Jasmin

Lam, Alan Tin-Lun

Lam, Yan

Lamatsch, Dunja

Lambert, Gilles

Lamichhane, Santosh

Lamm-Shalem, Noa

Lampert, Kathrin

Lanciotti, Angela

Lande, Roberto

Landfield, Philip W.

Lang, Di
Langguth, Peter

Langsley, Gordon

Lanzarone, Giuseppe

Lapucci, Andrea

Laskaratos, Faidon-Marios

Laskowska, Marzena

Lasseaux, Eulalie

Lässer, Cecilia

Lassot, Iréna

Latowski, Dariusz

Lautenschlaeger, Tim

Layer, Liliana

Lazarević, Vladimir

Lázaro-Diéguez, Francisco

Lazzaro, Federico

Le, Hoang-Thanh

Lebensztejn, Dariusz Marek

Lebiedzinska-Arciszewska, Magdalena

Lechel, Andre

Lechniak, Dorota

Leclerc, Estelle

Lecoq, Lauriane

Lederkremer, Gerardo Z.

Ledesma, Juan Carlos

Lee, Cheok Soon

Lee, Chia-Hwa

Lee, Dong Ryul

Lee, Geun-Shik

Lee, Hankyu

Lee, Ho

Lee, Jae

Lee, Jeong-Chae

Lee, Ji Hyun

Lee, Juhyun

Lee, Kyung-Ah

Lee, Li-Ang

Lee, Min Young

Lee, Ming-Jen

Lee, Myeongsang

Lee, Sang-Han

Lee, Seung Hwan

Lee, Sooyeon

Lee, Stella

Lee, Sue C.

Lee, Sung Hoon

Lee, Sung Sik

Lee, Sung-Jae

Lee, Sunjae

Lee, Taesic

Lee, Wai-Leng

Lee, Wan 
Lee, Wen-Chin

Lee, Yun-Sil

Lee, Zhenghong

Lee-Sarwar, Kathleen Anne

Lefevre, Sophie D.

Lefrançois, Stéphane

Legembre, Patrick

Legendre, Florence

Legewie, Stefan

Legrand, Fanny

Legrand, Sylvain

Lehmann, Helge Immo

Lehotsky, Jan

Lei, Wei

Leinonen, Ville

Leis, Jonathan P.

Leitch, Harry

Lemcke, Heicko

Lemieux, Hélène

Lemonnier, Loic

Lennikov, Anton

Lenzi, Paola

Leo, Damiana

Leon Serrano, Javier

Leon, Juan

Leonardo, Costantino

Leondaritis, George

Leoni, Valerio

Leppik, Liudmila

Lerman, Lilach

Lerner, Ulf

Leskovac, Andreja R.

Leszczuk, Agata

Leu, Jiann-Horng

Leung, Anthony K. L.

Leung, George P. H.

Levin, Leonard

Levin, Lonny R.

Levitsky, Dmitrii

Lewalle, Philippe

Lewicki, Sławomir

Lewis, Tylor R.

Lezot, Frederic

Li Puma, Domenica Donatella

$\mathrm{Li}, \mathrm{Bo}$

Li, Chien-Feng

Li, Fengzhi

Li, Guangzhao

Li, Jiao Jiao

$\mathrm{Li}$, Jun

Li, Ka Wan
Li, Li

Li, Po-Hsien

Li, Shang

$\mathrm{Li}$, Shengwen Calvin

Li, Tianhu

Li, Wei

Li, Wenbo

Li, Yafeng

Li, Yan

Li, Yi Shuan Julie

Li, Yutian

Li, Ziru

Liakopoulos, Dimitris

Liampas, Ioannis

Lian, Qizhou

Liao, Chengheng

Liao, Peng

Libert, Claude

Lichtenauer, Michael

Lie, Dieter Chichung

Liebau, Stefan

Liedert, Astrid M.

Liepins, Janis

Liepman, Aaron

Lifjeld, Jan T.

Lilly, Mary

Lim, Chin Yan

Lim, Jaechul

Lim, Lee Wei

Lim, Sang-Moo

Lim, Tingsen Benson

Lim, Wonbong

Lim, Yunping

Limoli, Paolo

Lin, Bin

Lin, Chih-Hsun

Lin, Chih-Jen (Lance)

Lin, Chih-Li

Lin, Chingyu

Lin, Ho

Lin, Jer-An

Lin, Li

Lin, Qing

Lin, Sheng-Chieh

Lin, Yung-Kai

Linard, Christine

Lindenberg, Svend

Lindner, Holger A.

Lindquist, Jonathan

Lindsay, Andrew J.

Lindstedt, Sandra 
Liobikas, Julius

Lionetti, Vincenzo

Lipinski, Patryk

Lipinski, Pawel

Lira, Fábio Santos

Lirussi, Frédéric

Lisachov, Artem P.

Lisby, Michael

Lisowska, Katarzyna

Lisowska, Katarzyna M.

Liu, Aimin

Liu, Ai-Ming

Liu, Chenying

Liu, Chia-Chi

Liu, Chun

Liu, Jian-Min

Liu, Luning

Liu, Michelle Tianqing

Liu, Mingyao

Liu, Ning

Liu, Pengda

Liu, Ping-Yen

Liu, Shing-Hwa

Liu, Xiangsheng

Liu, Yaling

Liu, Yen-Liang

Liu, Yi-Chang

Liu, Yie

Liu, Yi-Wen

Liu, Yongfeng

Liu, Yonggang

Liu, Yu-Peng

Liu, Zhenlong

Ljubimov, Alexander V.

Llansola, Marta

Lleo, Mar

Llobet, Artur

Llorens Martin, Maria

Llorens-Bobadilla, Enric

Lloyd, R. Stephen

Lo Gullo, Alberto

Lo Nigro, Luca

Lo, Chunmin C.

Lo, Michael

Lobachev, Kirill

Lobachevsky, Pavel

Lobo, Glenn

Lobo, María Val Toledo

Lochhead, Jeffrey J.

Locker, Felix

Lodola, Francesco
Loeffler, Jean-Philippe

Loiola, Rodrigo A.

Lokstein, Heiko

Lombardi, Raffaella

Lombó, Marta

Lonardi, Sara

Lonardo, Amedeo

Lonardo, Enza

Loo, Shining

Lopes, Coeli M. B.

Lopes, Marilene Hohmuth

López Malo, Daniel

Lopez, Blanca R.

López, Claudia S.

Lopez, Jose Javier

López, Yair

López-Alemany, Roser

Lopez-Camarillo, Cesar

López-Moya, Juan José

Lopitz Otsoa, Fernando

Loppin, Benjamin

Loppini, Alessandro

LoPresti, Patrizia

Lorenzl, Stefan

Lorenzo González, Óscar

Loreto, Andrea

Lossius, Andreas

Lotz, Christopher

Lou, Yan-Ru

Louis, Sandrine

Love, Seth

Low, Lucie

Lu, Huiming

Lu, Richard

Lu, Wan-Jung

Lu, Wenying

Lu, Yi-Chien

Lu, Yujiao

Lualdi, Marta

Lubrano, Valter

Luca, Giovanni

Luci, Carmelo

Luciani, Giovanni Battista

Lucien, Fabrice

Luddi, Alice

Ludidi, Ndiko

Luduena, Richard

Ludvigsen, Maja

Luganini, Anna

Luissint, Anny Claude

Lukaszewicz-Zajac, Marta 
Lukáts, Ákos

Lukomska, Ewa

Lund, Elizabeth K.

Lunder, Mojca

Lüpold, Stefan

Lushington, Gerry

Łuszczki, Jarogniew

Lux, Christopher

Luxton, G. W. Gant

Lyck, Ruth

Lyng, Fiona

Ma, Hansong

Ma, Hongming

Ma, Suk Ling

Ma, Wen-Juan

Määttä, Jorma A.

Macauley, Matthew S.

Maccarinelli, Federica

MacDiarmid, Robin

Mace, Maria L.

Macek Jilkova, Zuzana

MacEwan, David

Machado, Isidro

Machairiotis, Nikolaos

Machin, Daniel

Maclaczyk, Jaroslaw

Mackenzie, Louise S.

Mackiewicz-Milewska, Magdalena

MacLean, Andrew G.

Madamsetty, Vijay Sagar

Madaro, Luca

Madduri, Srinivas

Madka, Venkateshwar

Madkour, Aicha

Madoui, Mohammed-Amin

Madrigal, Irene

Madureira, Patrícia A.

Maeda, Kazuhiko

Mafalda, Santos

Magadum, Ajit

Magalon, Guy

Magaye, Ruth R.

Maggi, Leonard B.

Maggiolini, Marcello

Magi, Simona

Magin, Thomas

Magnaghi, Valerio

Magnan, Bruno

Magne, David

Magnelli, Lucia

Magrassi, Lorenzo
Magrì, Andrea

Mahajan, Gautam

Maia, Cláudio

Mailleux, Arnaud

Maina, Flavio

Maioli, Margherita

Maiorano, Domenico

Maiques-Diaz, Alba

Maixent, Jean Michel

Maj, Malgorzata

Majima, Hideyuki J.

Majumder, Pritha

Makishima, Makoto

Malagolini, Nadia

Malamitsi-Puchner, Ariadne

Malapelle, Umberto

Malashicheva, Anna

Malfa, Giuseppe

Malfatti, Edoardo

Malik, Anna R.

Mallard, Beth

Mallela, Shamroop Kumar

Malli, Roland

Mamouni, Kenza

Mamun-Or-Rashid, A. N. M.

Mancini, Maicol

Mancuso, Salvatrice

Mandl, Markus

Mandolesi, Georgia

Manera, Umberto

Manes, Nathan P.

Manetti, Mirko

Manferdini, Cristina

Manfredi, Francesco

Manganelli, Valeria

Mangino, Giorgio

Mani, Chinnadurai

Mani, Sridhar

Manirujjaman, M.

Manivel, Vivek

Manna, Prasenjit

Mannion, Arlene

Manole, Emilia

Manzano, Raquel

Manzo, Emiliano

Manzoor, Rashid

Manzotti, Gloria

Mao, Xiao Wen

Mapelli, Lisa

Maraldi, Tullia

Maranesi, Margherita 
Marasco, Wayne

Marazziti, Daniela

Marcello, Elena

Marcenaro, Emanuela

Marchi, Saverio

Marcia, Marco

Marconi, Guya Diletta

Maréchal, Alexandre

Marei, Hany

Margolis, Leonid

Margulis, Boris

Maria, Sirakov

Marian, Brigitte

Marin, Philippe

Marina, Anabel Isabel

Marini, Herbert Ryan

Marini, Patricia Estela

Marino, Joseph

Marjomaki, Varpu

Markiewicz, Aleksandra

Markuszewski, Michał

Marone, Gianni

Maroteaux, Luc

Marques, André R. A.

Marques, Isabel

Marrano, Nicola

Marrelli, Massimo

Marsch-Martinez, Nayelli

Marselli, Lorella

Marsh, Leigh M.

Marshall, Lindsay

Marteyn, Antoine

Martin, Daniel

Martin, Elizabeth C.

Martín, Margarita

Martín-Cófreces, Noa B.

Martínez Contreras, Rebeca Debora

Martínez De Pablos, Rocío

Martínez, Miguel A.

Martinez, Pedro

Martínez-Núñez, Mario Alberto

Martínez-Rodríguez, Gonzalo

Martínez-Sánchez, Noelia

Martinez-Useros, Javier

Martini, Andrea

Martini, Claudia

Martini, Emmanuelle

Martins, Mariana Renovato

Martín-Vasallo, Pablo

Martí-Sistac, Octavi

Marullo, Stefano
Maruyama, Junki

Maruyama, Satoshi

Marx, Christian

Marzec, Michal

Masarone, Daniele

Masola, Valentina

Massa, Valentina

Massányi, Peter

Massaro, Fulvio

Massaro, Giulia

Massart, Julie

Massie, Ann

Mastropasqua, Leonardo

Masuzaki, Ryota

Máthé, Csaba

Mathieu, Coureuil

Mathieu, Julie

Mathis, Bryan J.

Mathonnet, Muriel

Matsubara, Shigeki

Matsubara, Shin

Matsubara, Takuma

Matsuda, Yoshiko

Matsuo, Kazuya

Matsuzaki, Junko

Matt, Stephanie

Mattei, Vincenzo

Matthäus, Claudia

Matthews, Jason

Maturana, Carola J.

Maugeri, Alessandro

Maugeri, Grazia

Maurits, Elmer

Maury, Wendy

Mauvezin, Caroline

Mauvoisin, Daniel

Mavrogianni, Vasia S.

Mawhinney, Thomas

Maycotte, Paola

Mayer, Arnulf

Mayerhofer, Artur

Mazur, Antonina Joanna

Mazzawi, Tarek

McAlinden, Kielan Darcy

McCartney, Stephen A.

McCaughan, Geoff

McClements, Lana

McCourt, Peter

McDonough, Ashley

McIntosh, J. Richard (Dick)

Mckay, Tina 
McKeown, Lynn

McKimmie, Clive

McLean, Mary

McMillin, Matthew

Meabon, James S.

Mead, Ben

Meana, Clara

Means, Robert T.

Mears, Jason

Meccariello, Rosaria

Mechoulam, Raphael

Mederacke, Ingmar

Medert, Rebekka

Medica, Davide

Medina, Kay L.

Medina-Rodríguez, Eva M.

Medrano, Francisco J.

Mees, Maarten

Megraw, Timothy L.

Mehterov, Nikolay

Meijer, Dies

Meinhardt, Matthias

Mekori, Yoseph

Meleppattu, Shimi

Meler, Eva

Melgaço, Juliana G.

Mellitzer, Georg

Mello, Maria Luiza S.

Melloni, Elisabetta

Melo, Rossana

Menanteau-Ledouble, Simon

Menchaca, Jorge Luis

Mencke, Rik

Méndez Bolaina, Enrique

Mendez, Aaron

Mendoza-Almanza, Gretel

Menè, Paolo

Meng, Guangxun

Menis, Jessica

Menna, Elisabetta

Menon, Manoj B.

Merchán, Miguel Ángel

Merchant, Juanita

Merdes, Andreas

Merino, Beatriz

Merle, Philippe

Meroni, Germana

Merrick, William C.

Mersaoui, Sofiane Y.

Mert, Karakaya

Mertens, Jerome
Merwid-Ląd, Anna

Mesonero, José E.

Mesquita, Thássio

Metere, Alessio

Methner, Axel

Metzinger, Laurent

Meyer Zu Heringdorf, Dagmar

Meyer, Irene

Meyerhans, Andreas

Mezey, Eva

Mi, Lan

Miano, Maria Giuseppina

Micha, Dimitra

Michaelsen-Preusse, Kristin

Micheli, Fabienne

Michelon, William

Middei, Silvia

Miedema, Jelle R.

Miele, Adriana E.

Migaud, Marie

Migliaccio, Antimo

Migliaccio, Christopher

Mihály, József

Mika, Delphine

Mikoč, Andreja

Mikula, Mario

Milanesi, Elena

Mildner, Michael

Milena, Rondón-Lagos

Miliauskas, Skaidrius

Milisav, Irina

Miller, Karl

Miller, Yury I.

Miller-Kasprzak, Ewa

Miloshev, George

Milthorpe, Bruce

Minafra, Luigi

Minea, Radu

Minelli, Alessandro

Minematsu, Takeo

Minguela, Alfredo

Miniero, Roberto

Minutolo, Antonella

Miranda, Catarina

Miranda, Elena

Mirisola, Mario

Mishra, Aarti

Mishra, Hemant K.

Mishra, Jay S.

Misiak, Błazej

Missale, Gabriele 
Mistriotis, Panagiotis

Mitchell, Cassie S.

Mitchison, Timothy

Mititelu Tartau, Liliana

Mitola, Stefania

Mitra, Sumonto

Mitsuaki, Moriyama

Mitton, Kenneth

Miura, Shinji

Miyamoto, Ikuya

Miyata, Jun

Miyazaki, Kouji

Miyoshi, Keiko

Mizrachi, Dario

Mizuno, Mitsuru

Mizushima, Noboru

Mlynarska, Agnieszka

Mo, Fei

Mobasheri, Ali

Mobed-Miremadi, Maryam

Moby, Vanessa

Mochizuki-Kashio, Makiko

Moeinzadeh, Seyedsina

Moha Ou Maati, Hamid

Mohamed, Tamer

Mohammad, Khalid

Mohan, Ramkumar

Mohapatra, Debananda

Mohibi, Shakur

Mohsen, Mona

Mohseny, Alexander B.

Mohsin, Sadia

Mok, Chee Keng

Moles, Jean-pierre

Molina Holgado, Eduardo

Molina, Cristina E.

Mollace, Vincenzo

Mollapour, Mehdi

Molnár, Elek

Momekov, Georgi

Moncal, Kazim K.

Mondal, Ashis

Monje-Casas, Fernando

Monné, Magnus

Monsalves-Alvarez, Matias

Montalbano, Mauro

Montana, Giovanna

Montecucco, Fabrizio

Montemurro, Nicola

Montes, Nuria

Monzani, Fabio
Monzen, Satoru

Moon, Eun-Yi

Moon, Jisook

Moon, Minho

Moon, Seung Myung

Moraczewska, Johanna

Morais-Silva, Gessynger

Morales, Albert

Morales, Julio

Morales, Olivier

Morales, Serafin

Morales-González, José Antonio

Morales-Sánchez, Abigail

More, Shyam

Moreau, Violaine

Moreira, Daniel Carneiro

Moreira, Paula

Moreira, Vanessa

Moreira-Filho, Carlos Alberto

Moreno Loshuertos, Raquel

Moreno, Estefanía

Moreno, Sandra

Moreno-Andrés, Daniel

Moretti, Marta

Morfoisse, Florent

Morgan, Michael

Morganti, Claudia

Morgese, Maria Grazia

Mori, Yasuko

Morichi, Shinichiro

Morimoto, Tatsuya

Morimura, Shigeru

Moriondo, Andrea

Moris, Arnaud

Moriscot, Anselmo

Moriscot, Anselmo Sigari

Morleo, Manuela

Moroncini, GianLuca

Morozov, Alexey V.

Moser, Simone

Mosienko, Valentina

Mosiołek, Anna

Motterlini, Roberto

Mottet, Denis

Mou, Hongmei

Mou, Yongchao

Mounier, Catherine

Moustogiannis, Athanasios

Mravec, Boris

Msallam, Rasha A.

Muceniece, Ruta 
Mueller, Yvonne

Mukai, Tomoyuki

Mukherjee, Rukmini

Mukhtar, Mahwash

Mukohda, Masashi

Muldoon, Timothy J.

Mulens-Arias, Vladimir

Mulet, Jose M.

Mulherkar, Shalaka

Muller, Patricia A. J.

Müller, Thomas

Muniraj, Nethaji

Muñoz Pinedo, Cristina

Muñoz, Ana

Muñoz, Juan Pablo

Münsterberg, Andrea

Munteanu, Cristian V. A.

Munyard, Kylie A.

Murakami, Kosaku

Muraro, Elena

Murase, Daiki

Murayama, Takashi

Muriel, Pablo

Murín, Radoslav

Murín, Radovan

Murooka, Thomas

Murphy, Ronan

Murray, Rachael

Murthy, Divya

Musgrave, Ian

Mustelin, Tomas

Mustonen, Anne-Mari

Mus-Veteau, Isabelle

Mutsaers, Steven E.

Muzza, Marina

Myatt, Leslie

Myklebust, June Helen

Myllykallio, Hannu

Mylonis, Ilias

Myung, Jihwan

Nabe, Takeshi

Nabissi, Massimo

Nadal, Alfons

Nadal-Nicolas, Francisco

Nádasy, György

Nagai, Maria

Nagaoka, Satoshi

Nagasaka, Kazunori

Nagendran, Jayan

Nagre, Nagaraja

Naik, Akshata
Najle, Sebastián Rodrigo

Nakada-Tsukui, Kumiko

Nakae, Jun

Nakagawa, Takayuki

Nakagomi, Takayuki

Nakajima, Hideaki

Nakajima, Kei

Nakamichi, Noritaka

Nakamura, Takafumi

Nakamura-Ishizu, Ayako

Nakano, Nobuhiro

Nakase, Hiroshi

Nakayama, Katsutoshi

Nakayama, Masafumi

Nakazawa, Mitsuru

Nakchbandi, Inaam

Nalepa, Irena

Nalvarte, Ivan

Nam, Young-jae

Namba, Fumihiko

Nambeesan, Savithri

Namikawa, Kazuhiko

Nampoothiri, Madhavan

Nan, Xiaolin

Nanako, Kawaguchi

Nandurkar, Harshal

Napoli, Pietro

Narbonne, Patrick

Nardozza, Simona

Narra, Sudhakar

Naryzhny, Stanislav N.

Nasarre, Patrick

Nashine, Sonali

Nass, Norbert

Nassa, Giovanni

Nassir, Fatiha

Nasso, Giuseppe

Nastasi, Claudia

Nat, Irina Roxana

Natvig, Donald O.

Nauli, Andromeda M.

Nava, Sara

Navarrete, Marcelo A.

Navarro, Estanis

Navarro, Juan Antonio

Naves, Thomas

Nawrot, Barbara

Nawrotek, Paweł

Nayak, Nihar Ranjan

Naziroglu, Mustafa

Nechaev, Sergei 
Neelamegham, Sriram

Neill, Stewart G.

$\mathrm{Nel}$, Ivonne

Nelson, Leonard J.

Németh, Attila

Neuhauss, Stephan

Neumann, Detlef

Neupane, Yub Raj

Neureiter, Daniel

Nevler, Avinoam

Nguyen, Isabel

Nguyen, Paul

Nguyen, Thi-Huong

$\mathrm{Ni}$, Wei

Nicholas, Ekow Thomford

Nicita, Francesco

Nicoletta, Barbani

Nieland, John Dirk Vestergaard

Niello, Marco

Niemelä, Onni J.

Niemeyer, Barbara A.

Niesor, Eric J.

Nii, Teruki

Niibe, Kunimichi

Niikawa, Hiromichi

Nijakowski, Kacper

Nikitopoulou, Ioanna

Nikolaev, Viacheslav

Nikoloudakis, Nikolaos

Nikovics, Krisztina

Niño-Castro, Andrea Cecilia

Nir, Uri

Nirengi, Shinsuke

Nishida, Kensei

Nishimune, Hiroshi

Nishimura, Ken

Nissinen, Anne I.

Nitika, Nitika

Nobs, Samuel Philip

Nocella, Cristina

Nofer, Jerzy-Roch

Nógrádi, Antal

Nogueira, Fernando

Nohales, Maria A.

Nonoguchi, Hiroshi

Noothalapati, Hemanth

Noren Hooten, Nicole

Noro, Rintaro

North, Brian J.

Nosi, Daniele

Noureini, Sakineh Kazemi
Novopashina, Darya

Novotny, Jiri

Ntanasis-Stathopoulos, Ioannis

Nucera, Eleonora

Nugraha, Bramasta

Numakawa, Tadahiro

Nuñez, Angel

Nunzio, Vicario

Nurjadi, Dennis

Oberg, Kerby C.

Obeso, David

Obinata, Daisuke

Ocampos, Fernanda Maria Marins

Ochi, Shinichiro

Ochiai, Daigo

Ochiai, Kazuhiko

Ochoa-Callejero, Laura

Ocker, Matthias

O'Connell, Kevin F.

Oczkowski, Michał

Odierna, Gaëtano

Offermann, Anne

Ogiwara, Katsueki

Ogorek, Rafal

Oh, Sekyung

O'Hara, Steven

Ohbayashi, Norihiko

Ohbuchi, Toyoaki

Ohe, Joo-Young

Ohishi, Tomokazu

Ohshiro, Takahito

Ohtaka-Maruyama, Chiaki

Ohya, Susumu

Ohyama, Ayako

Ojha, Shreesh K.

Oka, Tomonori

Okabe, Seiichi

Okada, Shigeru

Okajima, Tetsuya

Okamoto, Mariko

Okazaki, Yasumasa

Okbay, Aysu

O'Keefe, Louise

Okła, Karolina

Oksenych, Valentyn

Oláh, Judit

Olah, Marta

Olaru, Octavian Tudorel

Olchawa, Magdalena M.

Oleari, Roberto

Oliva, Joan 
Olivares-Reyes, Jesús Alberto

Oliveira, Karla Mychellyne Costa

Oliveira, Raquel

Oliver, Lisa

Olivero, Antonella

Olmo, Ettore

Olsen, Abby L.

Olson, Michael

Omarjee, Loukman

Omichinski, James G.

Ondřej, Vladan

Onizuka, Satoru

Ono, Katsushige

Ono, Ryuichi

Ono, Yasuko

Onoda, Yusuke

Onofrillo, Carmine

Onori, Paolo

Ontsouka, Edgar

Oota, Keiichi

Opazo, Patricio

Opiela, Jolanta

Opoka, Wlodzimierz

Ordonez-Moran, Paloma

Orellana, Esteban

Orengo, Dorcas

Orinska, Zane

Orlandini, Maurizio

Orlov, Yuri

Orozco, Luis

Ortega, Angel L.

Ortega-Villasante, Cristina

Ortin-Martinez, Arturo

Ortín-Martínez, Arturo

Ortis, Fernanda

Ortiz, Angelíca

Ortiz-Masiá, Dolores

Osada, Shin-Ichi

Osmanagic-Myers, Selma

Osmulski, Pawel A.

Osredkar, Joško

Osses, Nelson

Ostacolo, Carmine

Ostasov, Pavel

O'Sullivan, Jamie

Oswald, Franz

Otsuki, Takemi

Ott, Maria

Ottaviano, Margaret

Otto, Angela

Ottoboni, Linda
$\mathrm{Ou}, \mathrm{C}$. C.

Ouzzine, Mohamed

Ozaki, Shuji

Ozretić, Petar

Pacary, Emilie

Pacheco, Yves

Pacherník, Jiří

Pacia, Marta Z.

Pacifici, Roberto

Pacurari, Maricica

Padler-Karavani, Vered

Pagano, Sabrina

Pałasz, Artur

Palazón-García, Ramiro

Palikaras, Konstantinos

Palleschi, Alessandro

Pallotti, Francesco

Palma, Paulo J.

Palmer, Donald

Palmerini, Maria

Palomares, Oscar

Palumbi, Roberto

Palyulin, Vladimir

Pan, Chun-Hsu

Pan, Jiayi

Pan, Ming-Kai

Panaro, Maria Antonietta

Pandey, Ashutosh

Pandey, Sudhir

Pandit, Anjali

Panetta, Daniele

Panizzutti, Bruna

Pannaccione, Anna

Pantopoulos, Kostas

Panzarini, Elisa

Panzitt, Katrin

Pao, Ping-Chieh

Papa, Giovanni

Papaccio, Federica

Papaccio, Gianpaolo

Papadaki, Chara

Papadaki, Maria

Papadakis, Georgios E.

Papaioannou, Andriana I.

Papait, Andrea

Papareddy, Ranjith K.

Papini, Alessio

Paplinska, Magdalena

Paponov, Ivan A.

Papp, Bela

Papp, Bernadett 
Parackova, Zuzana

Paradowska-Gorycka, Agnieszka

Parameswaran, Neetha

Parekh, Anant

Parimon, Tanyalak

Parini, Angelo

Park, Daeho

Park, Eun Jeong

Park, Han-A

Park, Jae-Hyung

Park, Jeongho

Park, Jong Kook

Park, Joon Ha

Park, Ki-cheong

Park, Sophie

Park, Yong-Jin

Parker, Matthew F. L.

Parlatescu, Ioanina

Parma, Pietro

Parmar, Mayur

Parolini, Isabella

Paronetto, Maria Paola

Parra-Damas, Arnaldo

Parrilla, Inmaculada

Parrotta, Elvira

Parrozzani, Raffaele

Pascual, Gloria

Pasek, Jarosław

Passaro, Fabiana

Pasto, Anna

Pastorek, Jaromir

Patankar, Jay V.

Patel, Kapil D.

Patel, Nibedita

Patel, Vaibhav B.

Patenge, Nadja

Patergnani, Simone

Patnaik, Sourav

Patriarca, Andrea

Patrussi, Laura

Patsoukis, Nikolaos E

Pattappa, Girish

Pattnaik, Bikash

Paudel, Keshav Raj

Paudel, Pradeep

Paullada Salmerón, José Antonio

Paul-Visse, Gesine

Pause, Arnim

Pautz, Andrea

Pauwels, Laurens

Pávek, Petr
Pavlova, Elena

Paw, Milena

Pawelczyk, Tadeusz

Pawlikowski, Marek

Pawlina, Klaudia

Payne, Annette

Payre, François

Pazgan-Simon, Monika

Peake, Nicholas

Pecqueur, Claire

Pedemonte, Nicoletta

Pedone, Elisa

Peh, Hong Yong

Peinado, María Ángeles

Pejler, Gunnar

Pelidou, Sygkliti-Henrietta

Pellet-Many, Caroline

Pellicciari, Maria

Pendin, Diana

Penfornis, Patrice

Peng, I-Chen

Penke, Botond

Penke, Loka

Penn, Raymond

Penning, Louis

Penson, Peter

Pentimalli, Francesca

Peperzak, Victor

Peppelenbosch, M. P.

Peraçoli, Maria Terezinha Serrão

Peraldi, Pascal

Peralta, Carmen

Perazzoli, Gloria

Perdigones, Nieves

Perego, Carla

Pereira Ferrer, Valeria

Pereira, Leonel

Pereira, Patrícia Alexandra

Perek, Barłłomiej

Peres, Lazaro Eustaquio Pereira

Perez Del Pulgar, Sofia

Pérez, Antonio

Pérez-Campo, Flor María

Pérez-Rubio, Gloria

Periasamy, Muthu

Periasamy, Ramesh

Pericàs, Juan M.

Perini, Marcos

Perišić, Ognjen

Pernet, Vincent

Peronnet, Frederique 
Perret, Alain

Perrine-Walker, Francine

Perris, Roberto

Perucca Orfei, Carlotta

Perucci, Luiza O.

Pesovic, Jovan

Pestov, Dimitri

Peterson, Eliza J. R.

Pethő, Gábor

Petit, Patrice X.

Petkov, Stoyan

Petrak, Jiri

Petrenko, Volodymyr

Pettit, Kristen

Peyronnet, Rémi

Pfitzner, Emanuel

Phan, Thi Tuong Vy

Piancone, Federica

Piao, Wenji

Piastowska-Ciesielska, Agnieszka

Picart, Thiebaud

Piccionello, Antonio Palumbo

Piccioni, Andrea

Piccirillo, Rosanna

Piccoli, Marco

Pickering, Raelene

Picketts, David J.

Picone, Pasquale

Pieretti, Stefano

Pierno, Sabata

Pieroni, Maurizio

Pietras, Alexander

Pietras, Eric

Pievani, Alice

Piffoux, Max

Pileczki, Valentina

Pileri, Alessandro

Pilette, Charles

Piliponsky, Adrian

Pimienta, Rodney Lacret

Pinart, Elisabeth

Pineda, José R.

Pinheiro, Paulo S.

Pink, Daniel

Pinós, Tomás

Pinto, José Renato

Pinto, Luisa

Pinto, Maria Joana

Piperi, Christina

Pirkmajer, Sergej

Pisarev, Vladimir
Pisciotta, Alessandra

Piscitelli, Fabiana

Pissarek, Margit

Pistello, Mauro

Pistis, Marco

Pistocchi, Anna Silvia

Pittalà, Valeria

Piubelli, Chiara

Piva, Francesco

Pizzato, Massimo

Platt, Jeffrey L.

Platta, Harald W.

Playford, Martin P.

Plaza-Zabala, Ainhoa

Pleniceanu, Oren

Plotegher, Nicoletta

Plotnikov, Egor

Plotton, Ingrid

Pluta, Ryszard

Po, Agnese

Pocheć, Ewa

Poillet-Perez, Laura

Póka, Róbert

Pol, Jonathan

Polacheck, William

Polakowski, Nicholas J.

Polańczyk, Andrzej

Polge, Cécile

Polverino De Laureto, Patrizia

Poma, Adolfo

Poma, Anna

Pomierny-Chamioło, Lucyna

Pomorski, Paweł

Poncet, Delphine

Pongratz, Georg

Ponte, Nuno

Pontes, Bruno

Poon, Ivan

Poór, Péter

Pop, Laurentiu

Popa-Wagner, Aurel

Popescu, Bogdan O.

Popova, Blagovesta

Popper, Helmut H.

Porat-Shliom, Natalie

Porciatti, Vittorio

Pors, Klaus

Porta, Helena

Porteu, Françoise

Porzio, Elena

Post, Mark 
Potaczek, Daniel P.

Potes, Yaiza

Potokar, Maja

Potrony, Miriam

Pouget, Pierre

Poulios, Stylianos

Pouliot, Roxane

Poulsen, Kyle

Poupot, Remy

Poyner, David

Pozzo, Federico

Pradet-Balade, Berengere

Pradhan, Dinesh

Pradhan, Sriharsa

Prakash, Hridayesh

Prakash, Nilima

Pramanik, Arindam

Prantl, Lukas

Prasad, Kasavajhala

Prêle, Cecilia M.

Prestileo, Tullio

Preta, Giulio

Priante, Giovanna

Priel, Avi

Prieto, Rafael Maria

Prigent, Claude

Primožič, Ines

Pritchard, Michele

Pritesh, Jain

Privette Vinnedge, Lisa Privette

Prochowska, Sylwia

Procopio, Francesco Andrea

Prodromou, Chrisostomos

Proescholdt, Martin

Prokop, Jeremy W.

Prondzynski, Maksymilian

Prosperi, Jenifer

Protchenko, Olga

Provost, Patrick

Pruijm, Menno T. C.

Przybyło, Małgorzata

Pucci, Fabrizio

Pucino, Valentina

Puglisi, Rossella

Pujia, Arturo

Pullen, Nicholas

Pulliero, Alessandra

Puniya, Bhanwar Lal

Purchase, Craig

Pusch, Stefan

Püschel, Gerhard P.
Pushchina, Evgeniya

Pustylnyak, Vladimir

Puthenparampil, Marco

Puukila, Stephanie

Qasim, Muhammad

Qi, Xiaoyang

Qi, Yue

Qian, Yanrong

Qu, Lili

Qu, Ning

Quaglino, Daniela

Quax, Paul

Querol Audí, Jordi

Quesada, Antonio

Quintanilla, Rodrigo A.

Raasakka, Arne

Rabelink, Ton

Racaud-Sultan, Claire

Rachek, Lyudmila I.

Racioppi, Luigi

Raczkowska, Joanna

Radælli, Enrico

Rademaker, Marius

Radenkovic, Miroslav

Radinger, Madeleine

Radisavljevic, Ziv

Radosavljević, Tatjana

Radu, Beatrice Mihaela

Rageul, Julie

Raggi, Paolo

Raghav, Pawan Kumar

Raghunath, Michael

Ragni, Enrico

Ragusa, Michael J.

Rahaghi, Franck Farzad

Rahman, M. Azizur

Rahman, Masmudur M.

Rai, Vikrant

Raimondi, Lavinia

Raimondo, Diego

Rajagopal, Sudarshan

Rajamohan, Arun

Rajendran, Peramaiyan

Rajendran, Ramya Lakshmi

Rajendren, Suba

Rakic, Pasko

Rakonczay, Zoltán

Ralvenius, William

Ramachandra, Chrishan

Ramadori, Giuliano

Ramalho-Santos, João 
Ramírez Sebastián, Ana I.

Ramos, Ana Paula

Ramos, Carlos

Ramprasath, Tharmarajan

Ramsden, David

Ramsköld, Daniel

Ranganna, Kasturi

Rangel, Luciana Pereira

Ranieri, Girolamo

Ranjit, Sabina

Ranzato, Elia

Rao, Mangala

Rapino, Cinzia

Rappeneau, Virginie

Rasin, Mladen Roko

Rašin, Mladen Roko

Rasmussen, Lene

Rasola, Andrea

Rassow, Joachim

Rastegar, Mojgan

Rasul, Sazan

Ratajczak, Mariusz Z

Rathinasabapathy, Anandharajan

Rato, Luís

Ratti, Stefano

Ratushnyy, Andrey

Raut, Pawan Kumar

Ravaioli, Francesco

Ravasio, Andrea

Ravens, Sarina

Ravens, Ursula

Rayburn, Edward B.

Real, Luis Miguel

Rébé, Cédric

Recsan, Zsuzsanna

Reddy, Sangeetha

Redell, Michele S.

Reggiani, Carlo

Reggio, Alessio

Rehman, Attiq Ur

Reichardt, Holger M.

Reiff, Tobias

Reinach, Peter S.

Reindl, Wolfgang

Reineke, Lucas

Reiner, Orly

Reiss, Allison B.

Reitman, Marc

Relat, Joana

Relvas, Joao

Remmerswaal, Ester B. M.
Renaut, Jenny

Repetto, Marisa Gabriela

Resch, Ulrike

Reshkin, Stephan

Reshkin, Stephan Joel

Reuken, Philipp A.

Reutov, Valentin

Reuveni, Moshe

Revechon, Gwladys

Revuri, Vishnu

Reyes, José Luis

Reynaert, Niki L.

Režen, Tadeja

Rezoagli, Emanuele

Rezus, Elena

Rhee, Kunsoo

Ribas, Vicente

Ribeiro, Luís

Ribeyre, Cyril

Ribitsch, Iris

Ricci, Erika

Ricciotti, Emanuela

Richard, Bodnar

Richards, Allison

Richards, Dylan Jack

Richter, Günther H. S.

Richter, Vladimir

Ricklefs, Franz Lennard

Ridrigo, Ana

Riederer, Peter

Riera, Marta

Rigoni, Michela

Rijavec, Matija

Riku, Yuichi

Rim, Yeri

Rinnerthaler, Mark

Riobo, Natalia

Riou, Laurent M.

Ritz, Ulrike

Rius-Pérez, Sergio

Riuzzi, Francesca

Riva, Nilo

Rivera-Toledo, Evelyn

Rivero, Francisco

Rivero-Müller, Adolfo

Rivoltini, Licia

Rizzarelli, Enrico

Rizzi, Roberto

Rizzo, Alessandro

Ro, Hyunju

Robak, Tadeusz 
Robert, David

Robert, Philippe A.

Roberto, Michela

Robertson, Avril

Robertson, Shelly

Robin, François B.

Robinson, Mathew

Rocha, Emily M.

Rochaix, Jean-david

Roche, Sandra

Rochfort, Keith

Röder, Stefan

Roderburg, Christoph

Rodrigo, Luis

Rodrigue, Agnès

Rodrigues, Alice C.

Rodrigues, Fernando José Santos

Rodrigues, Pedro Miguel

Rodrigues, Teresa

Rodriguez, Anne-marie

Rodriguez, Rafael

Rodriguez, Raphaël

Rodríguez, Ricardo Reyes

Rodríguez, Víctor Manuel

Rodriguez-Blanco, Jezabel

Rodriguez-dorantes, Mauricio

Rodriguez-Fernandez, Jose Luis

Rodríguez-Kessler, Margarita

Rodríguez-Pallares, Jannette

Rodríguez-Vita, Juan

Rodríguez-Yoldi, Maríajesús

Roduit, Raphael

Roe, Judith L.

Roelen, Bernard

Roger, Lauréline

Rogers, Ian M.

Rog-Zielinska, Eva Alicja

Roh, Gu Seob

Roh, Sangho

Rohde, David

Rohlena, Jakub

Rojiani, Mumtaz V.

Rojo, Enrique

Rojo, Maria Angeles

Roma, Cristin

Romanel, Alessandro

Romanelli, Maria Grazia

Romani, Patrizia

Romaniello, Donatella

Romanin, Christoph

Romanini, Antonella
Romano, Arturo

Romano, Corrado

Romano, Giovanni Luca

Romano, Marco

Romano, Nicla

Romanyuk, Nataliya

Romei, Cristina

Romeo, Maria Anele

Romero, Marta R.

Romero-López, Cristina

Romero-Lopez, Monica

Rona, Roberto

Ronca, Roberto

Rondeau, Philippe

Rosado, Iván Valle

Rosado, Juan

Rosas-Vargas, Haydeé

Rosati, Elisa

Rosati, Jessica

Roşca, Ana Maria

Roscini, Luca

Rose, Alan B.

Rosenberg, Mai

Rosenspire, A. J.

Rosenthal, Cynthia

Rosenthal, Dean S.

Rosetti, Florencia

Rosi, Antonella

Rosi, Susanna

Rosina, Marco

Rossa, Carlos, Jr.

Rossi, Elisabetta

Rossi, Gianpaolo

Rossi, Rachele

Rossignol, Julien

Rosso, Chiara

Rossowska, Joanna

Rota, Paola

Rotblat, Barak

Roth, Steven

Rothhammer, Veit

Rotstein, Benjamin $\mathrm{H}$.

Rotti, Pavana

Rouleau, Matthieu

Roumenina, Lubka

Roux, Kyle

Rovatsos, Michail

Roy, Milton

Rozalski, Marcin

Rozanowska, Malgorzata

Rozentryt, Piotr 
Rozman, Primoz

Rozzo, Carla

Ruaro, Barbara

Rubelj, Ivica

Rubilotta, Emanuele

Rubino, Federico Maria

Rugonyi, Sandra

Rump, Katharina

Rund, Deborah

Rurali, Erica

Rusciano, Dario

Rusciano, Maria Rosaria

Rusek, Marta

Rusin, Marek

Rusmini, Paola

Russell, Oliver

Russo, Matteo A.

Russo, Tommaso

Ruzafa, Noelia

Ruzov, Alexey

Ruzza, Paolo

Ryazansky, Sergei

Rybska, Marta

Rybtsov, Stanislav

Ryczek, Natalia

Ryffel, Bernhard

Ryskalin, Larisa

Rzepka, Zuzanna

Rzymowska, Jolanta

Sabater, Lidia

Sabbatinelli, Jacopo

Sabbatini, Maria Eugenia

Sabol, Maja

Sacco, Elena

Saccone, Salvatore

Sacedón, Rosa

Sachadyn, Paweł

Sachinidis, Agapios

Sack, Ulrich

Sadagurski, Marianna

Sadasivam, Mohanraj

Sadeghi, Saeed

Sadhasivam, Balaji

Sadhukhan, Pritam

Sadoul, Karin

Saeki, Kumiko

Saeki, Toshiaki

Saez, Fabrice

Saéz, Juan Carlos

Sáfrány, Géza

Sagdullaev, Botir T.
Saglio, Giuseppe

Sah, Bert-Ram

Sah, Nirnath

Saha, Shekhar

Saha, Sushanta Kumar

Sahgal, Pranshu

Said, Elias A.

Saint-Pol, Julien

Sainz, Bruno

Saisho, Yoshifumi

Saito, Takashi

Sakai, Ken

Sakai, Shinji

Sakamoto, Atsushi

Sakamoto, Yuzuru

Saksena, Nitin

Sakwe, Amos

Sala, Claudia

Sala, Mariaelvina

Salamon, Dominika

Salehi, S. Albert

Salinas, Eva

Salman, Mootaz

Salomé Pires, Ana

Salton, Francesco

Salvadori, Susanna

Salvatorelli, Emanuela

Salvetti, Anna

Samaddar, Madhuja

Samaja, Michele

Samarzija, Ivana

Samasca, Gabriel

Sambri, Andrea

Šamec, Dunja

Samec, Marek

Samidurai, Arun

Sampaio-Marques, Belém

Sampetrean, Oltea

Samuchiwal, Sachin K.

Sánchez, Aránzazu

Sánchez-Mendoza, Alicia

Sánchez-Pernaute, Rosario

Sandoval-Acuña, Cristián

Sandrim, Valéria Cristina

Saneyasu, Takaoki

Sanfeliu, Coral

Sang, Yongming

Sangadala, Sreedhara

Sangiolo, Dario

Sankaran, Vijay G.

Santaeufemia, Sergio 
Santamaria-Martínez, Albert

Santarelli, Andrea

Santhoshkumar, Puttur

Santinon, Giulia

Santos, Joana M. O.

Santos, Mariana

Santulli, Gaetano

Sanz, Carmen

Sanz, Pascual

Sapienza, Daniela

Saraiva, Miguel Mascarenhas

Sarantseva, Svetlana

Sardanelli, Anna Maria

Saretzki, Gabriele

Sarkar, Arijita

Sarkar, Gobinda

Sarkar, Susobhan

Sarma, Pranjal

Sarnowska, Elzbieta

Sastre, Leandro

Sato, Priscila

Sato, Takeshi

Satou, Ryousuke

Šatović, Eva

Saucedo-García, Mariana

Sauma, Daniela

Savary, Stéphane

Savatier, Pierre

Savica, Rodolfo

Savio, Monica

Sawicka, Emilia

Saxena, Vikas

Sayed, Ahmed

Sayed, Ibrahim M.

Sazonova, Margarita A.

Scalia, Rosario

Scarano, Aurelia

Scarpa, Fabio

Scarpelli, Paolo

Scarpellini, Emidio

Scattolini, Valentina

Scaturro, Dalila

Schäfer, Katrin

Schallmoser, Katharina

Schemmer, Peter

Schiedat, Fabian

Schierwagen, Robert

Schildhaus, Hans-Ulrich

Schirhagl, Romana

Schirmer, Bastian

Schirone, Leonardo
Schlecht-Louf, Géraldine

Schlegel, Patrick

Schlein, Christian

Schlereth, Simona L.

Schlüter, Klaus-Dieter

Schmaier, Alvin H.

Schmetterer, Klaus

Schmid, Nina

Schmidl, Doreen

Schmidt, Martina

Schmidtke, Gunter

Schmitz, Thomas

Schneider, Keith

Schneider-Maunoury, Sylvie

Schneitz, Kay

Scholte, Florine

Schoot, Reineke A.

Schreml, Stephan

Schrock, Morgan

Schröder, Agnes

Schröder, Henrik Daa

Schroeder, Anna

Schrottmaier, Waltraud C.

Schryvers, Anthony

Schubert, Frank Richard

Schubert, Ulrich

Schueler, Julia

Schuler, Herwig

Schulze, Harald

Schulze-Hentrich, Julia

Schulze-Osthoff, Klaus

Schulze-Tanzil, Gundula Gesine

Schumacher, Anne

Schuurmans, Carol

Schuyler, Scott C.

Schwarzer, Roland

Schwenkenbecher, Philipp

Sciandra, Francesca

Scicchitano, Stefania

Scimone, Concetta

Scorpio, Diana

Scupoli, Maria Teresa

Sebastian, Robin

Šeda, Ondřej

Sedgewick, Jerry

Sedykh, Sergey

Seethala, Raja R.

Segatto, Marco

Seger, Rony

Segu, Marzia

Segundo, Fayna Diaz-San 
Sehgal, Deepmala

Seibler, Philip

Seifalian, Alexander

Seiler, Magdalene J.

Seiliez, Iban

Sellal, Jean-Marc

Selzer, Michael E.

Semczuk, Andrzej

Semenyuk, Pavel

Sempere, Lorenzo

Sen, Monokesh Kumer

Senetta, Rebecca

Senis, Yotis

Seo, Jae-Hong

Seracchioli, Renato

Seralini, Gilles-Eric

Serbanovic-Canic, Jovana

Sereno, Denis

Sereti, Evangelia

Serge, Plaza

Sergeeva, Olga

Seroogy, Kim B.

Serra, Aida

Serra, Maria Pina

Serrador Peiró, Juan M.

Serra-Moreno, Ruth

Serrano, Emmanuel

Serrano-Mollar, Anna

Serrano-Pozo, Alberto

Serratì, Simona

Sesillo, Francesca Boscolo

Sessa, Francesco

Sestito, Rosanna

Sethi, Gautam

Sevastre, Bogdan

Ševčík, Jan

Severino, Paolo

Sfikakis, Petros P.

Sgarbanti, Marco

Shah, Vaibhav

Shahbaaz, Mohd

Shahbazi, Marta N.

Shakibaei, Mehdi

Shalaby, Sarah

Shanely, R. Andrew

Shao, Wen Hai

Sharakhov, Igor

Sharif, Jafar

Sharma, Dipali

Sharma, Jaiprakash

Sharma, Tripti
Sharoar, Md. Golam

Shashikanth, Nitesh

Shaul, Yoav D.

Shaul, Yosef

Shaykhiev, Renat

Shearer, Cameron

Shekhova, Elena

Shen, ChangHui

Shen, Haihong

Shepherd, Trevor

Sheth, Bhavwanti

Shevach, Ethan

Shi, De-Li

Shi, Haoshen

Shi, Jiajun

Shi, Jian

Shi, Patricia A.

Shiba, Kiyotaka

Shibata, Yasushi

Shie, Ming-You

Shigdar, Sarah

Shih, Yin-Hwa

Shiina, Nobuyuki

Shim, Jae-Hyuck

Shim, Joon W.

Shimada, Yohta

Shimakawa, Ginga

Shimaoka, Motomu

Shimizu, Takatsune

Shimizu, Taro

Shimokawa, Naofumi

Shin, Crystal S.

Shin, Kichul

Shinozaki, Yoshihito

Shirahata, Tatsuya

Shirakawa, Hitoshi

Shizu, Ryota

Shortland, Peter

Shu, Xinhua

Shukla, Kirtikar

Shvetsova, Anastasia A.

Sibille, Nathalie

Sierra-Campos, Erick

Siewert, Katherina

Silachev, Denis

Silva, Diana

Silva, Nuno A.

Silva, Severino Jefferson Ribeiro

Silver, Frederick H.

Silvestre, Miguel Angel

Silvestre-Roig, Carlos 
Simić, Tatjana

Simoes, Davina Camargo Madeira

Simon, Hans-uwe

Simone, Jonathan

Simos, George

Simpson, Julie E.

Since, Marc

Sinenko, Sergey A.

Singh, Karmveer

Singh, Kasha Priya

Singh, Meenesh R.

Singh, Nisha

Singh, Shashi Shekhar

Singh, Sitanshu S.

Singh, Zeba Niazi

Sini, Maria Cristina

Sinigaglia, Alessandro

Sinigaglia, Chiara

Šinko, Goran

Sioofy-Khojine, Amirbabak

Sioud, Mouldy

Sipeki, Nóra

Sipos, Katalin

Sirico, Domenico

Sirois, Martin G.

Siska, Peter J.

Sisto, Francesca

Sitzia, Clementina

Sivakumar, Sushama

Skinner, Pamela

Skladany, Lubomir

Skok, Maryna

Skonieczna-Żydecka, Karolina

Skuli, Nicolas

Skup, Malgorzata

Skuratovskaia, Daria

Skuza, Lidia

Slaaby, Rita

Slade, Neda

Sławińska, Urszula

Sleigh, James

Slijepcevic, Predrag

Śliwiński, Tomasz

Slominski, Andrzej

Slovin, Susan

Smedowski, Adrian

Smeland, Sigbjørn

Śmigiel, Robert

Smith, Gary D.

Smythe, Elizabeth

So, Jae-Seon
Soares, Sylvia

Soba, Peter

Sobinoff, Alexander

Sobrido-Cameán, Daniel

Sodeik, Beate

Sofinska, Kamila

Sohrabi, Yahya

Soilleux, Elizabeth

Soldan, Samantha S.

Soldati, Gianni

Soler, Carles

Solomonov, Inna

Solovchenko, Alexei

Sommer, Grün Nicole

Song, Dong-Keun

Song, Jaewhan

Song, Jun-Ho

Song, Rui

Song, Wenxia

Song, Ziyi

Soni, Chetna

Sonntag, Kai

Sontake, Vishwaraj

Soontorngun, Nitnipa

Sordi, Valeria

Sorelli, Marcela

Soreq, Hermona

Sorg, Olivier

Soria, Federico N.

Soriano-Romaní, Laura

Soriano-Sarabia, Natalia

Sorice, Maurizio

Sorio, C.

Sorokin, Vitaly A.

Sorokina, Oksana

Sorrentino, Raffaella

Sorrentino, Vincenzo

Sortino, Maria Angela

Soshnikova, Natalia

Sosic, Izidor

Sossin, Wayne

Soulika, Athena

Soundararajan, Meera

Sousa, Mónica Mendes

Souyri, Michele

Spagnuolo, Carmela

Spampinato, Santi

Sparagna, Genevieve C.

Sparrer, Konstantin M. J.

Spartalis, Michael D.

Speake, Cate 
Spears, Norah

Spencer, Peter S.

Sperdouli, Ilektra

Spike, Benjamin T.

Spinelli, Laura

Spite, Matthew

Spletter, Maria

Sposi, Nadia Maria

Squatrito, Massimo

Šrámek, Jan

Srivastava, Akriti

Sriwastva, Mukesh Kumar

Staal, Frank J. T.

Stacchiotti, Alessandra

Stahl, Mirjam

Stančiaková, Lucia

Standal, Therese

Stanescu-Spinu, Iulia-Ioana

Stanislawska-Sachadyn, Anna

Stanke, Frauke

Stark, Lesley A.

St-Arnaud, Rene

Starobova, Hana

Starzyński, Rafał

Stathopulos, Peter

Stauber, Tobias

Staudinger, Jeff L.

Stayner, Cherie

Stecco, Carla

Steenbrugge, Jonas

Stefan, Simm

Steffen, Imke

Štefulj, Jasminka

Steinman, Gary

Stellos, Konstantinos

Stenmark, Kurt

Stepanov, Alexey V.

Stephens, Jacqueline M.

Stępniewski, Jacek

Stevens, Claire H.

Stevenson-Lerner, Heather

Stewart, Jason

Stewart, Sarah

Stewart-Ornstein, Jacob

Stimpfel, Martin

Stinghen, Andréa Emilia Marques

Stival, Cintia

Stocco, Carlos

Stokin, Gorzard Bernard

Storchi, Loriano

Stott, Jennifer B.
St-Pierre, Marie V.

Strafella, Claudia

Stratmann, Bernd

Stratton, Richard

Straube, Jasmin

Strazhesko, Irina

Stricker, Sigmar

Strosznajder, Joanna B.

Štrumfa, Ilze

Strzelecka-Kiliszek, Agnieszka

Stuart, David T.

Stukenborg, Jan-Bernd

$\mathrm{Su}$, Chih-Chia

Su, Qiaozhu

Subhi, Yousif

Subramanian, Sowmyalakshmi

Sudol, Marius

Sueyoshi, Tatsuya

Sugawara, Akira

Sugawara, Shigeki

Sugaya, Makoto

Sugii, Hideki

Sugimoto, Michelle A.

Suhito, Intan Rosalina

Sul, Jai-Yoon

Suleiman, M.-Saadeh

Suliman, Hagir B.

Sullivan, Con

Sullivan, Patrick G.

Sun, Alfred Xuyang

Sun, Xiaodong

Sun, Xingmin

Sun, Ye

Sun, Yin

Sunter, Jack D.

Surapaneni, Krishna Mohan

Surdo, Nicoletta Concetta

Surman, Magdalena

Surugiu, Victor

Suryadevara, Vidyani

Sutanto, Henry

Sutherland, James D.

Sutter, Andreas

Suzuki, Aussie

Suzuki, Kazuhito

Suzuki, Susumu

Suzuki, Yuichiro J.

Svalbe, Baiba

Svensson, Mattias N. D.

Svirskis, Simons

Świątkiewicz, Iwona 
Swiech, Kamilla

Syed, Naweed

Sylow, Lykke

Szablewski, Leszek

Szabo, Ildiko

Szabó, Renáta

Szakiel, Anna

Szanto, Magdolna

Szatmari, Erzsebet Maria

Szczepankiewicz, Aleksandra

Szczepańska, Maria

Szczepek, Agnieszka J.

Szeberény, József

Szekeres, Mária

Szeri, Flóra

Szewczyk, Adam

Szibor, Marten

Szigeti, Kinga

Szoke, Eva

Szokodi, István

Szulcek, Robert

Szumera-Cieckiewicz, Anna

Szychowski, Konrad A.

Taanman, Jan-Willem

Tabarkiewicz, Jacek

Tabbò, Fabrizio

Taboada-Serrano, Patricia

Taha, Zaid

Tahirovic, Sabina

Taija, Mäkinen

Taillandier, Daniel

Tailleux, Anne

Takabatake, Kiyofumi

Takahashi, Chiaki

Takahashi, Toshio

Takam Kamga, Paul

Takeda, Shunichi

Takeuchi, Jun K.

Talaverón, Rocío

Tamaian, Radu

Tamarit, Jordi

Tamba, Bogdan Ionel

Tamkovich, Svetlana

Tampa, Mircea

Tan, Tse-Hua

Tan, Tuan Zea

Tanaka, Hiroyoshi Y.

Tanaka, Miwa

Tanaka, Nobuyuki

Tanaka, Satoshi

Tanaka, Takuji
Tancredi, Virginia

Tandon, Ritesh

Tang, Anna

Tang, Chih-Yung

Taniguchi, Hiroaki

Taniguchi, Masaaki

Tanner, Jessie C.

Tanos, Barbara

Tantos, Ágnes

Tao, Junyan

Tao, Zhipeng

Tao-Cheng, Jung-Hwa

Taouis, Mohamed

Tarallo, Valeria

Tarantino, Giovanni

Tarantino, Umberto

Tarasov, Andrei

Tartey, Sarang

Taru Sharma, Gutulla

Tasaki, Osamu

Taschler, Ulrike

Tasken, Kjetil

Tassieri, Manlio

Tateno, Toru

Tátrai, Péter

Tattini, Massimiliano

Tavian, Daniela

Tawk, Marcel

Taylor, Andrew W.

Taymans, Jean-Marc

Tchoghandjian-Auphan, Aurélie

Teague, Heather L.

Teasdale, Rohan

Teijeiro, Juan Manuel

Teixeira, José H.

Teixidó, Cristina

Teneva, Ivanka

Teodoro, João

Teper, Sławomir Jan

Teraa, Martin

Terao, Mineko

Terentyev, Vasily V.

Terlizzi, Michela

Terry, Cassandra

Tesar, Vladimir

Tesarik, Jan

Tessem, Jeffery S.

Testi, Anna Maria

Testoni, Barbara

Tews, Birke

Thakur, Shilpa 
Thangavel, Chellappagounder

Thankam, Finosh G.

Theoharides, Theoharis C.

Theopold, Ulrich

Theotokis, Paschalis

Theret, Marine

Thevenot, Paul T.

Thiam, Hawa Racine

Thielemann, Christiane

Thiem, Alexander

Thierry, Alain

Thomas, Charles

Thongprayoon, Charat

Thorne, Peter

Thorsby, Per Medbøe

Tian, Xiaoyu

Tiberio, Laura

Tilocca, Bruno

Timmins, Joanna

Timofeev, Sergey

Tissier, Paul Le

Tixeira, Rochelle

Toapanta, Franklin R.

Tochigi, Yuki

Toczek, Jakub

Todorović, Ana

Todorovic, Slobodan

Todt, Daniel

Toffan, Anna

Togashi, Marie

Togashi, Tatsuya

Toh, Wei Seong

Toietta, Gabriele

Tokarek, Tomasz

Tokunaga, Fuminori

Tolkacheva, Alena G.

Tolwinski, Nicholas

Tomar, Dhanendra

Tomasello, Marianna Flora

Tomaszewska, Ewa

Tomov, Martin L.

Tomuleasa, Ciprian

Tonazzini, Ilaria

Tonelli, Michele

Tong, Qingyi

Tong, Xin

Toporowska, Magdalena

Torgasheva, Anna

Törnquist, Kid

Torras, Juan

Torres, Raul
Torsello, Antonio

Toscani, Denise

Toshimitsu, Yamaoka

Tossetta, Giovanni

Tosti, Elisabetta

Toth, Rachel

Totolian, Areg

Totta, Pierangela

Touil-Boukoffa, Chafia

Toyoda, Masashi

Traiffort, Élisabeth

Traina, Giovanna

Traini, Tonino

Traka, Maria

Tramutola, Antonella

Tran, Hai Bac

Tranbarger, Timothy John

Tranquilli Leali, Paolo Tranquilli

Tranter, Michael

Trapero, Isabel

Treglia, Giorgio

Trendelenburg, Marten

Trentini, Alessandro

Tribouillard-Tanvier, Déborah

Tribulova, Narcis

Tricarico, Domenico

Trifilieff, Pierre

Triggiani, V.

Trincavelli, Maria Letizia

Tringali, Stephane

Trinkaus-Randall, Vickery

Tripathi, Ashutosh

Troadec, Jean-Denis

Troadec, Marie-Bérengère

Troncoso-Ponce, Adrian

Trouche, Didier

Trouillas, Jacqueline

Trounson, Alan

Truchado, Marta

Trzcińska, Monika

Tsagareli, Merab G.

Tsagozis, Panagiotis

Tsai, Kun-Lin

Tsai, Rongkung

Tsaniklidis, Georgios

Tsikou, Daniela

Tsimokha, Anna S.

Tsou, Wei Ling

Tsuchiya, Hiroyuki

Tsuge, Tomohiko

Tsukamoto, Sho 
Tsukuba, Takayuki

Tůma, Zdeněk

Tungjai, Montree

Tura-Ceide, Olga

Turu, Gábor

Tuttolomondo, Antonino

Tutusaus, Anna

Tuyaerts, Sandra

Tvrda, Eva

Tvrdá, Eva

Twumasi Osei, Emmanuel

Tyler, Jessica

Tylzanowski, Przemko

Tyrkalska, Sylwia

Tyukmaeva, Venera I.

Tzanoudaki, Marianna

Tzen, Jason T. C.

Tzoneva, Rumiana

Uchil, Pradeep D.

Uddin, Md Jamal

Ueda, Jun

Ueno, Hiroshi

Ulasov, Ilya

Ullah, Ghanim

Ullevig, Sarah

Ulvmar, Maria H.

Um, Ji Won

Umansky, Samuil

Umbarkar, Prachi

Umehara, Mikihisa

Umemura, Naoki

Ungar, Daniel

Ungurianu, Anca

Unternaehrer, Juli

Uphoff, Stephan

Uras, Iris Z.

Urbańska, Ewa M.

Urciuolo, Anna

Uroš, Andjelković

Usaj, Marko

Ushimaru, Takashi

Usta, Osman

Usui, Tatsuya

Uysal-Onganer, Pinar

Uziel, Orit

Vaccaro, Maria

Vafiadaki, Elizabeth

Vagnozzi, Ronald

Vago, Juliana

Valcke, Roland

Valdés-Baizabal, Catalina
Valdez-Jasso, Daniela

Valenti, Maria

Valenzuela, Rita

Valiño-Rivas, Lara

Valjent, Emmanuel

Valkov, Eugene

Vallejo, Alejandro

Valles, Soraya L.

Vamanu, Emanuel

Van De Worp, Wouter R. P. H.

Van Den Broek, Daan

Van Der Horst, Gerhard

Van Der Kuyl, Antoinette

Van Der Laan, Martin

Van Der Vorst, Emiel

van Dievoet, Marie-Astrid

Van Eijk, Marco

Van Ginderachter, Jo

van Gisbergen, Klaas P. J. M.

Van Gisbergen, Marike W.

Van Grevenynghe, Julien

Van IJzendoorn, David

Van Maele, Laurye

Van Nieuwenhoven, Frans A.

Van Ry, Pam

Van Soom, Ann

Van Vliet, Kim M.

Van Wuytswinkel, Olivier

Van Zonneveld, Anton Jan

Vandekerckhove, Bart

Vanden Heuvel, Gregory B.

Vandevoorde, Charlot

Vandewalle, Jolien

Van-Dijk, Juliette

Vandooren, Jennifer

Vanneaux, Valérie

Vannini, Eleonora

Vannucchi, Maria-Giuliana

Vaqué, José Pedro

Váradi, Csaba

Varani, Gabriele

Varga, Elisabeth

Varin, Audrey

Varju, Cecilia

Varna-Pannerec, Mariana

Varricchio, Ettore

Vaschetto, Rosanna

Vasileva, Liliya

Vatter, Hartmut

Vaucher, Elvire

Vaverka, Miroslav 
Vavvas, Demetrios G.

Vaz, Ana Rita

Vaz, Raquel

Vazquez, Elba S.

Vecino, Elena

Vedunova, Maria V.

Vega Alvarez, Jose Antonio

Vega, Francisco M.

Vega-Benedetti, Ana Florencia

Velardi, Enrico

Velasco, Iván

Velayutham, Murugesan

Velazquez, Laura

Velentzas, Athanassios D.

Velepic, Marko

Velikova, Tsvetelina

Veltkamp, Marcel

Venetucci, Luigi

Venkatesan, Shriram

Vennerstrom, Jonathan

Ventura, Célia

Vercoutter-Edouart, Anne-Sophie

Verdurmen, Wouter P. R.

Vergadi, Eleni

Vergara, Daniele

Vermiglio, Giovanna

Verna, Roberto

Veroux, Massimiliano

Verrecchia, Franck

Verri, Tiziano

Verrier, Eloi R.

Versino, Elisabetta

Verticchio, Alice

Vetter, Marcus

Veyron-Churlet, Romain

Vicari, Marcelo Ricardo

Vicario-Abejón, Carlos

Vicaș, Laura Grațiela

Vicente, Cristina P.

Vicente, Giner Galván

Viczian, Andras

Vida, Carmen

Vidal, Rene

Vidal-Sanz, Manuel

Vieyra, Adalberto

Viggiano, Emanuela

Vijayaanand, Arokia Mariyadoss

Vijayakumar, Sarath

Vilaboa, Nuria

Villareal, Myra

Villas-Boas, Gustavo R.
Vincent, C. Theresa

Vincent, Delphine

Vincentini, Olimpia

Vinci, Cristina

Vincze, Eva

Virag, Laszlo

Visentin, Sergio

Vishnoi, Kanchan

Vishweswaraiah, Sangeetha

Visioli, Francesco

Vitetta, Luis

Vitorino, Rui

Vives, Joaquim

Vizoso-Vázquez, Angel

Vlachonasios, Konstantinos

Vlassi, Metaxia

Vodenkova, Sona

Vodickova, Ludmila

Voidarou, Chrissoula

Volkmer, Hansjürgen

Volonte, Cinzia

Volta, Francesco

Von Appen, Alexander

Von Hörsten, Stephan

Von Kopylow, Kathrein

Von Strandmann, Elke Pogge

Vontas, John G.

Vorselaars, Adriane D. M.

Vos, Melissa

Vrachatis, Dimitrios A.

Vrzal, Radim

Vuillefroy De Silly, Romain

Vuilleumier, Nicolas

Vukicevic, Slobodan

Vukojevic, Katarina

Vulpis, Elisabetta

Vydra, Natalia

Wachten, Dagmar

Wade, Rachael M.

Wadham, Carol

Waghray, Avinash

Wagner, Kay-Dietrich

Wahli, Walter

Wähnert, Dirk

Wainman, Alan

Wakimoto, Hiroaki

Walczak, Claire

Walczak-Drzewiecka, Aurelia

Walker, Douglas G.

Wallings, Rebecca L.

Walter, Fruzsina 
Walter, Silke

Walter, Ulrich

Walton, Richard

Wan, Lei

Wandinger-Ness, Angela U.

Wang, Aline Yen Ling

Wang, Bingyan J.

Wang, Chihyang

Wang, Chrong-Reen

Wang, Chun-Wei

Wang, Fun-In

Wang, Guan

Wang, Hsiuying

Wang, Hui-Chun

Wang, Hung-Jen

Wang, Jaw-Yuan

Wang, Jian-Ying

Wang, Jin'An

Wang, Jinghua

Wang, Jinli

Wang, Lan-Hsin

Wang, Lei

Wang, Ling

Wang, Lizhong

Wang, Nan-kai

Wang, Nifei

Wang, Peng

Wang, Richard

Wang, Shaobin

Wang, Shumin

Wang, Tong-Hong

Wang, Xiaofeng

Wang, Xiaoxin

Wang, Xuejun

Wang, Yajing

Wang, Yang-Kao

Wang, Yigang

Wang, Ying

Wang, Ying-Hsiu

Wang, Yuan-Hsi

Wang, Zhaoning

Wang, Zhaoqi

Wang, Zhixiang

Wang, Zhoufei

Wang, Zongjie

Want, Muzamil Yaqub

Ward, W. Steven

Waszkiewicz, Napoleon

Watanabe, Eiichi

Watanabe, Masakatsu

Watanabe, Toshio
Watanabe, Yasuo

Watkins, Paul A.

Wawrzyniak, Paulina

Wax, Adam

Wculek, Stefanie K.

Weaver, Tyler M.

Weber, K. Scott

Weeden, Norman F.

Wehrens, Xander H. T.

Wei, Jun

Wei-Cheng, Tseng

Weinberger, Florian

Weisberg, Ellen

Weiss, Étienne

Weiss, Martin

Weissenhorn, Winfried

Welner, Robert S.

Welsh, Michael

Wende, Wolfgang

Wendt, Lisa

Werneburg, Sebastian

Werner, Haim

Werner, Sabine

Wernersson, Sara

Wernly, Bernhard

Wesołowska, Olga

Westmark, Cara J.

Weston, Stuart

Wetzel, Scott

Wever, Alyssa

Wheeler, Dave

Wheeler, Michael

White, Fletcher

Whiteside, Eliza

Wibowo, Erik

Wichmann, Christian

Widemann, Emilie

Widiapradja, Alexander

Wiedlocha, Antoni

Wiegers, Gerrit-Jan

Wielockx, Ben

Wienkoop, Stefanie

Wigle, Jeffrey

Wijnholds, Jan

Wikonkál, Norbert

Wikström, Mårten

Wilber, Andrew C.

Wilhelm, Jochen

Wille, Holger

Willerth, Stephanie

William Richardson, William 
Williams, David

Willis, Dianna E.

Wilm, Matthias

Wing, Simon

Winsz-Szczotka, Katarzyna

Winter, Jochen

Wiszniewska, Alina

Witalisz-Siepracka, Agnieszka

Witarski, Wojciech

Witherden, Deborah

Witkiewicz, Wojciech

Włodarczyk, Marcin

Włoga, Dorota

Wnuk, Agnieszka

Wolf, Ido

Wolff, Daniel

Wołkow, Paweł

Won, Seok Joon

Wong, Boon-Seng

Wong, Chi-Ming

Wong, Chun Kwok

Wong, Chung

Wong, Dennis T. L.

Wong, Ka-Leung

Wong, Richard W.

Woo, Junsung

Woo, Se Joon

Woo, So-Youn

Wood, Thomas R.

Woodgett, Jim R.

Wright, Peter T.

Wsół, Agnieszka

$\mathrm{Wu}, \mathrm{Ai}-\mathrm{Min}$

$\mathrm{Wu}$, Bo-Sen

Wu, Chia Shan

Wu, Chun-Chi

Wu, Guangyu

$\mathrm{Wu}$, Huang-Pin

$\mathrm{Wu}$, Jianjun

$\mathrm{Wu}$, Lijun

Wu, Minghua

$\mathrm{Wu}$, Min-Huan

Wu, Qihui

Wu, Ray-chang

Wu, Shi-Bei

Wu, William

$\mathrm{Wu}$, Xiaoan

Wu, Yuh-Lin

Wuebbles, Ryan

Wyns, Christine

Wysokińska, Anna
Xavier, Stephenne

Xia, Guangbin

Xiao, Li

Xiao, Lu

Xiao, Yongli

Xiaoyan, Hui

Xie, Lai-hua

Xie, Lingxiao

Xie, Zhiqun

Xie, Zhongqiu

Xie, Zuoxu

Xin, Mei

Xing, D. A.

Xiong, Tou-Cheu

Xirodimas, Dmitris

$\mathrm{Xu}$, Jialin

$\mathrm{Xu}$, Jinbin

Xu, Kui

$\mathrm{Xu}$, Ren

$\mathrm{Xu}$, Suowen

Xu, Xuehua

$\mathrm{Xu}$, Yang

$\mathrm{Xu}$, Yan-Ming

Xue, Feng

Xue, Min

Yagi, Hiroshi

Yaish, Mahmoud

Yakhine-Diop, Sokhna M.S.

Yam, Gary Hin-Fai

Yamada, Takashi

Yamada, Taketo

Yamamoto, Akihito

Yamamoto, Masato

Yamamoto, Naoki

Yamaoka, Kunihiro

Yamaoka, Toshimitsu

Yamasaki, Tricia

Yamashita, Atsushi

Yamashita, Kimihiro

Yamashita, Toru

Yan, Bowen

Yan, Dayun

Yan, Huimin

Yanagisawa, Naoko

Yandeau-Nelson, Marna D.

Yang, Bo

Yang, Chuanbin

Yang, Chul-Su

Yang, Haining

Yang, Hung-Chi

Yang, Jiale 


\begin{tabular}{|c|c|}
\hline Yang, Kun & Yu, Xinfang \\
\hline Yang, Lili & Yu, Xinzhu \\
\hline Yang, Liu & Yuan, Meng \\
\hline Yang, Shun-Fa & Yuemeng, Jia \\
\hline Yang, Wen-Bin & Yumura, Shigehiko \\
\hline Yang, Xiulan & Yurchenko, Ekaterina \\
\hline Yang, Ying & Yutzey, Katherine \\
\hline Yang-Hartwich, Yang & Zaarour, Rania Faouzi \\
\hline Yanzhuang, Wang & Ząbek, Tomasz \\
\hline Yao, Herui & Zaboronok, Alexander \\
\hline Yao, Hiroshi & Zacchia, Miriam \\
\hline Yao, Jun & Zaccone, Giacomo \\
\hline Yao, Tao & Zadmajid, Vahid \\
\hline Yaparla, Amulya & Zadra, Giorgia \\
\hline Yarygin, Konstantin N. & Zaglia, Tania \\
\hline Yasuda, Takako & Zaid, Hilal \\
\hline Yazdi, Homa Papoli & Zaizen, Yoshiaki \\
\hline Ye, Jian & Zakłos-Szyda, Malgorzata \\
\hline Yen, Feng-Lin & Zakrocka, Izabela \\
\hline Yeo, Syn & Zalewska, Teresa \\
\hline Yerneni, Saigopalakrishna S. & Zalewska-Janowska, Anna \\
\hline Yesilkanal, Ali Ekrem & Zalvide, Juan \\
\hline Yévenes, Gonzalo & Zamfir, Carmen Lăcrămioara \\
\hline Yew, P. Renee & Zampieri, Stefania \\
\hline Yi, Chun-Xia & Zamyatnin, Andrey A. \\
\hline Yi, Joo Mi & Zandi, Peiman \\
\hline Ylösmäki, Erkko & Zanella, Isabella \\
\hline Ylostalo, Joni H. & Zanelli, Magda \\
\hline Yokota, Hiroki & Zanetti-Domingues, Laura C. \\
\hline Yong, Jeongsik & Zanfirescu, Anca \\
\hline Yoo, Yeong-Min & Zangle, Thomas \\
\hline Yoon, Hyeun Joong & Zapater, Pedro \\
\hline Yoon, Sung-Soo & Zapico, Sara Casado \\
\hline Yoon, Yisang & Zaramella, Patrizia \\
\hline Yoshida, Kaoru & Zarkovic, Neven \\
\hline Yoshimura, Kotaro & Zarnowski, Tomasz \\
\hline Yoshino, Hiide & Zarobkiewicz, Michał \\
\hline Yoshino, Hironori & Żarski, Daniel \\
\hline You, Hye Jin & Zatsepin, Timofei \\
\hline You, Jae-Sung & Zatz, Mayana \\
\hline Youm, Jae Boum & Zawadzka, Malgorzata \\
\hline Youn, Seock-Won & Zefferino, Roberto \\
\hline Young, Jessica & Zelarayan, Laura \\
\hline Yssel, Hans & Zelasco, Samanta \\
\hline Yu, Cheng-Chia & Żelechowska, Paulina \\
\hline Yu, Jia & Zemkova, Hana \\
\hline Yu, Jiashing & Zemła, Joanna \\
\hline Yu, Kai & Zenarruzabeitia, Olatz \\
\hline Yu, Shanping & Zeng, Baosheng \\
\hline Yu, Wei-Hsuan & Zeng, Bijun \\
\hline Yu, Wen-Chung & Zeng, Chang-Jun \\
\hline
\end{tabular}


Zeng, Shaohua

Zentilin, Lorena

Zerges, William

Zhang, Chun

Zhang, Dongyun

Zhang, Duo

Zhang, Hongquan

Zhang, Jin

Zhang, $\mathrm{Nu}$

Zhang, Qiuyang

Zhang, Wenjun

Zhang, Xiaonan

Zhang, Xingmin

Zhang, Yin Hua

Zhang, Yuan

Zhang, Yumin

Zhang, Zengdi

Zhao, Linlin

Zhao, Xianda

Zhao, Zhenjun

Zheng, Guoping

Zheng, Xiaoming

Zheng, Yun-Wen

Zhong, Quan

Zhong, Wei

Zhong, Zheng

Zhou, Bisheng

Zhou, Guangqian

Zhou, Jingsong

Zhou, Linli

Zhou, Meijuan

Zhou, Qingyu

Zhou, Shuanhu

Zhou, Xiaofeng

Zhou, Zhidong

Zhou, Zhifang

Zhu, Bangfu

Zhu, Dashuai
Zhu, Joe

Zhu, Mingzhao

Zhu, Qianzheng

Zhu, Wei

Zhu, Wuqiang

Zhuang, Shougang

Zhuang, Xiaohong

Zhuo, Chunliu

Žiarovská, Jana

Zidar, Nina

Ziegler, Tilman

Zieliński, Maciej

Ziemssen, Focke

Zikos, Panagiotis

Zíková, Martina

Zimmer, Jacques

Zimmer, Sebastian

Zimmer-Bensch, Geraldine

Ziolkowski, Wieslaw

Zissel, Gernot

Zivcak, Marek

Żołądek, Teresa

Zöller, Margot

Zondler, Lisa

Zong, Wei-Xing

Zórad, Stefan

Zordoky, Beshay

Zorec, Robert

Zornitsa Ivanova, Katerova

Zsigmond, Laura

Zubareva, Ekaterina V

Zubiaur, M.

Zundler, Sebastian

Zuo, Li

Župan, Ivan

Zupan, Janja

Zwaans, Bernadette M. M.

Zwart, Wilbert 